\title{
Transmuting off-shell CHY integrals in the double-cover framework
}

\author{
Kang Zhou ${ }^{\mathrm{a}}$, Guo-Jun Zhou ${ }^{\mathrm{b}}$ \\ Center for Gravitation and Cosmology, College of Physical Science and Technology, Yangzhou University, Yangzhou 225009, China
}

Received: 27 June 2020 / Accepted: 1 November 2020 / Published online: 19 November 2020

(C) The Author(s) 2020

\begin{abstract}
In this paper, by defining off-shell amplitudes as off-shell CHY integrals, and redefining the longitudinal operator, we demonstrate that the differential operators which link on-shell amplitudes for a variety of theories together link offshell amplitudes in a similar manner. Based on the algebraic property of the differential operator, we also generalize three relations among color-ordered on-shell amplitudes, including the color-ordered reversed relation, the photon decoupling relation, the Kleiss-Kuijf relation, to off-shell ones. The off-shell CHY integrals are chosen to be in the double-cover framework, thus, as a by product, our result also provides a verification for the double-cover construction.
\end{abstract}

\section{Introduction}

The Cachazo-He-Yuan (CHY) formalism reveals the marvelous unity among on-shell tree-level amplitudes of various theories [1-5]. In the CHY formulas, different theories correspond to different CHY integrands. Through the socalled dimensional reduction, squeezing, and the generalized dimensional reduction procedures, on-shell CHY integrands for a wide range of theories can be generated from the onshell CHY integrand for the gravity (GR) theory ${ }^{1}$ [5].

A similar unified set of on-shell tree-level amplitudes of different theories can be achieved via the differential operators proposed by Cheung, Shen and Wen. These differential operators, which act on Lorentz invariant kinematic variables, transmute the on-shell amplitude of one theory to that of another theory [6]. By applying these operators, the amplitudes for a variety of theories can be generated from the GR amplitude. The similarity between two unified sets implies

\footnotetext{
${ }^{1}$ In this paper the gravity theory is understood as the Einstein-Hilbert gravity coupled to a dilaton and two-forms.
}

a e-mail: zhoukang@yzu.edu.cn (corresponding author)

be-mail: zhou1750819726@163.com the underlying relationship between the two methods. This relationship has been spelled out in $[7,8]$, by acting differential operators on $\mathrm{CHY}$ integrals for different theories.

Based on the relations among on-shell amplitudes expressed by differential operators, several other relations for on-shell amplitudes can be derived. First, using the algebraic property of the differential operator, one can derive the generalized color-ordered reversed relation, the generalized photon decoupling relation, and the generalized Kleiss-Kuijf (KK) relation, which are valid for all color-ordered on-shell amplitudes [9]. Secondly, a dual version of the unified set, which reflects the relations among amplitudes by expanding the amplitude of one theory to amplitudes of another theory, can be established via the differential operators [9-11]. Notice that the expansions of the amplitudes have been studied from various points of view in the literature [12-21]. Among these, the approach used in [9-11] manifests the duality between the differential operators and the coefficients of the basis in the expansions.

In this paper, we demonstrate that the differential operators transmute off-shell amplitudes in a manner similar to that for on-shell amplitudes. The off-shell tree-level amplitudes in this paper are defined by off-shell CHY integrals. There are three motivations for considering off-shell amplitudes in the CHY framework. First, the massive external momentum with $k_{i}^{2} \neq 0$ can be treated as a special case of the off-shell massless momentum; thus, considering off-shell CHY integrals is an effective way to generalize the $\mathrm{CHY}$ formula to massive external states. Secondly, in the double-cover prescription proposed by Gomez, the evaluation of tree-level amplitudes in the CHY framework can be reduced to lowerpoint off-shell amplitudes, due to the factorizations realized by the double-cover method [22-27]. This is another important application of off-shell CHY integrals. Thirdly, from a theoretical point of view it is interesting to broaden the $\mathrm{CHY}$ formalism to reproduce off-shell tree-level amplitudes, and for loops to be computed, without a Lagrangian. 
The main method used in this paper is similar to that used in $[7,8]$, and can be summarized as follows. The off-shell treelevel amplitude in the $\mathrm{CHY}$ formula arises from the integral over auxiliary coordinates:

$\mathcal{A}_{n}=\int d \mu_{n} \mathcal{I}^{\mathrm{CHY}}$.

The measure part $d \mu_{n}$ is universal, while the CHY integrand $\mathcal{I}^{\mathrm{CHY}}$ depends on the theory under consideration. All the operators which will be considered in this paper act on Lorentz invariants $\epsilon_{i} \cdot \epsilon_{j}$ or $\epsilon_{i} \cdot k_{j}$, where $k_{i}$ and $\epsilon_{i}$ are the momentum and the polarization vector of the $i$ th leg, respectively. Thus, these operators will not affect the measure part which is independent of the polarization vectors. In other words, differential operators are commutable with the integral over auxiliary variables. Thus, transmuting an amplitude is equivalent to transmuting the corresponding CHY integrand. More explicitly, suppose $\mathcal{O}$ is a differential operator satisfying

$\mathcal{O} \mathcal{A}_{n}=\mathcal{O}\left(\int d \mu_{n} \mathcal{I}^{\mathrm{CHY}}\right)=\int d \mu_{n}\left(\mathcal{O I}^{\mathrm{CHY}}\right)$.

Thus, if two amplitudes $\mathcal{A}_{\alpha}$ and $\mathcal{A}_{\beta}$ are related by an operator $\mathcal{O}$ via $\mathcal{A}_{\alpha}=\mathcal{O} \mathcal{A}_{\beta}$; an analogous relation $\mathcal{I}_{\alpha}^{\mathrm{CHY}}=\mathcal{O} \mathcal{I}_{\beta}^{\mathrm{CHY}}$ for two integrands must hold, and vice versa. Consequently, one can derive the unifying relations systematically by applying differential operators to $\mathrm{CHY}$ integrands.

Using the above method, we will show that the unifying relations provided in [6], which link on-shell amplitudes of different theories, also hold for off-shell amplitudes defined by off-shell CHY integrals. There are three types of basic operators, the trace operator $\mathcal{T}[i, j]$, the insertion operator $\mathcal{I}_{i k j}$, and the longitudinal operators $\mathcal{L}_{i}$ and $\mathcal{L}_{i j}$, that will be considered in this paper. The definitions of the operators $\mathcal{T}[i, j]$ and $\mathcal{I}_{i k j}$ are the same as in [6-8], while the definitions of the longitudinal operators $\mathcal{L}_{i}$ and $\mathcal{L}_{i j}$ will be modified in the off-shell case. By applying the combinatory operators constructed by these three basic operators to the GR CHY integral, one can get the CHY integrals for theories including Einstein-Yang-Mills (EYM) theory, Yang-Mills (YM) theory, Einstein-Maxwell (EM) theory, Einstein-Maxwell theory with photons carrying flavors (EMf), Born-Infeld (BI) theory, Yang-Mills-scalar (YMS) theory, special Yang-Mills-scalar (sYMS) theory, bi-adjoint scalar (BAS) theory, the non-linear sigma model (NLSM), $\phi^{4}$ theory, Dirac-Born-Infeld (DBI) theory, extended DiracBorn-Infeld (exDBI) theory, and special Galileon (SG) theory. Since the unifying relations can be extended to offshell amplitudes, the relevant relations among color-ordered amplitudes, including the generalized color-ordered reversed relation, the generalized photon decoupling relation, and the generalized KK relation, can also be generalized to the offshell case.
In $[7,8], \mathrm{CHY}$ integrals are chosen to be in the original single-cover version. In this paper, we choose $\mathrm{CHY}$ integrals in the double-cover formulas [22-27]. Since on-shell amplitudes can be regarded as a special case of off-shell ones, if the unifying relations hold for off-shell amplitudes, they also hold for on-shell ones. Thus, our result in this paper provides a verification of the double-cover construction, because we have shown that the unifying relations for on-shell amplitudes in the single-cover formulas, which are already proved in $[7,8]$, are also correct for off-shell amplitudes in the doublecover framework.

The remainder of this paper is organized as follows. In Sect. 2, we give a brief introduction of the off-shell CHY formalism, and the double-cover prescription, which are necessary for subsequent discussions. In Sect. 3, we study the effects of three basic operators when applying them to building blocks of CHY integrands. In Sect. 4 we consider the effects of combinatory operators constructed by these basic operators. The relations among amplitudes, based on the previous preparations, are presented in Sect. 5. Finally, we end with a brief discussion in Sect. 6.

\section{Background}

For the reader's convenience, in this section we briefly review the off-shell CHY formalism, and the double-cover prescription.

\subsection{Off-shell CHY formalism}

The off-shell CHY formalism bears a strong similarity with the on-shell one, except the correction elements $\Delta_{i j}$ and $\eta_{i j}$ in scattering equations and matrix elements. In this subsection, we only introduce the off-shell CHY formalism. The on-shell one can be reproduced by taking $\Delta_{i j} \rightarrow 0, \eta_{i j} \rightarrow 0$.

In the off-shell CHY formula, the tree-level amplitude for $n$ massless particles arises from a multi-dimensional contour integral over the moduli space of genus zero Riemann surface with $n$ punctures, $\mathcal{M}_{0, n}$. It can be expressed as

$\mathcal{A}_{n}=\int d \mu_{n} \mathcal{I}_{L}\left(\left\{k_{i}, \epsilon_{i}, z_{i}\right\}\right) \mathcal{I}_{R}\left(\left\{k_{i}, \widetilde{\epsilon}_{i}, z_{i}\right\}\right)$,

which possesses the Möbius $\operatorname{SL}(2, \mathbb{C})$ invariance. Here $k_{i}$, $\epsilon_{i}$ (or $\tilde{\epsilon}_{i}$ ) and $z_{i}$ are the momentum, polarization vector, and puncture location for the $i^{\text {th }}$ external leg, respectively. The measure is defined as

$d \mu_{n} \equiv \frac{d^{n} z}{\operatorname{vol~SL}(2, \mathbb{C})} \frac{|p q r|}{\prod_{i=1, i \neq p q r}^{n} \mathcal{E}_{i}(z)}$. 
The factor $|p q r|$ is given by $|p q r| \equiv z_{p q} z_{q r} z_{r p}$, where $z_{i j} \equiv$ $z_{i}-z_{j}$. The off-shell scattering equations are given as $^{2}[28-$ 30]

$\mathcal{E}_{i}(z) \equiv \sum_{j \in\{1,2, \ldots, n\} \backslash\{i\}} \frac{2 k_{i} \cdot k_{j}+2 \Delta_{i j}}{z_{i j}}=0$.

These scattering equations satisfy the condition

$\sum_{i=1}^{n} z_{i}^{m} \mathcal{E}_{i}=0, \quad m=0,1,2$,

which protects the $\operatorname{SL}(2, \mathbb{C})$ invariance. This requirement imposes the constraint $\sum_{j \neq i} \Delta_{i j}=k_{i}^{2}$ on the elements $\Delta_{i j}$. For example, when $n \geq 5$, one can choose

$$
\begin{aligned}
\Delta_{i, i \pm 1} & =+\frac{1}{2}\left(k_{i}^{2}+k_{i \pm 1}^{2}\right), \\
\Delta_{i \mp 1, i \pm 1} & =-\frac{1}{2} k_{i}^{2}, \\
\Delta_{i j} & =0 \text { otherwise. }
\end{aligned}
$$

Here the cyclic symmetry of external legs has been used, for instance when $i=1$ we have $\Delta_{i, i-1}=\Delta_{1, n}, \Delta_{i-1, i+1}=$ $\Delta_{n, 2}$, and so on. Under the above choice, the scattering equations yield correct propagators in the Feynman gauge. The $(n-3)$ independent scattering equations define the map from the space of kinematic variables to $\mathcal{M}_{0, n}$, and fully localize the integral on their solutions. After fixing the $\operatorname{SL}(2, \mathbb{C})$ gauge, the measure part is changed to

$d \mu_{n} \equiv \frac{\left(\prod_{j=1, j \neq p, q, r}^{n} d z_{j}\right)|p q r|^{2}}{\prod_{i=1, i \neq p, q, r}^{n} \mathcal{E}_{i}(z)}$.

The integrand in (3) depends on the theory under consideration. For any theory known to have a $\mathrm{CHY}$ representation, the corresponding integrand can be factorized into two parts $\mathcal{I}_{L}$ and $\mathcal{I}_{R}$, as can be seen in (3). Either of them are weight-2 for each puncture coordinate $z_{i}$ under the $\operatorname{SL}(2, \mathbb{C})$ transformation. In Table 1, we list integrands for theories which will be considered in this paper ${ }^{3}$ [5].

We now explain each building block in turn. There are five kinds of $n \times n$ matrices, which are defined through

$$
\begin{aligned}
A_{i j} & = \begin{cases}\frac{k_{i} \cdot k_{j}+\Delta_{i j}}{z_{i j}} & i \neq j, \\
0 & i=j,\end{cases} \\
B_{i j} & = \begin{cases}\frac{\epsilon_{i} \cdot \epsilon_{j}}{z_{i j}} & i \neq j, \\
0 & i=j,\end{cases} \\
C_{i j} & = \begin{cases}\frac{k_{i} \cdot \epsilon_{j}+\eta_{i j}}{z_{i j}} & i \neq j, \\
-\sum_{l=1, l \neq j}^{n} C_{l i} & i=j,\end{cases}
\end{aligned}
$$

\footnotetext{
$\overline{2}$ In this paper, we choose $2 k_{i} \cdot k_{j}$ rather than $s_{i j}$ to define the scattering equations. Two choices are un-equivalent for off-shell momenta.

${ }^{3}$ For theories contain gauge or flavor groups, we only show the integrands for color-ordered partial amplitudes instead of full ones.
}

Table 1 Integrands for various theories

\begin{tabular}{lll}
\hline Theory & $\mathcal{I}_{L}\left(\left\{k_{i}, \epsilon_{i}, z_{i}\right\}\right)$ & $\mathcal{I}_{R}\left(\left\{k_{i}, \widetilde{\epsilon}_{i}, z_{i}\right\}\right)$ \\
\hline GR & $\mathbf{P f}^{\prime} \Psi$ & $\mathbf{P f}^{\prime} \Psi$ \\
EYM & $\mathcal{C}_{\operatorname{Tr}_{1}} \cdots \mathcal{C}_{\operatorname{Tr}_{m}} \sum_{\{i, j\}}{ }^{\prime} \mathcal{P}_{\{i, j\}}(n, l, m)$ & $\mathbf{P f}^{\prime} \Psi$ \\
YM & $\mathcal{C}_{n}(\sigma)$ & $\mathbf{P f}^{\prime} \Psi$ \\
EM & $\mathbf{P f}^{\prime}[\Psi]_{n-2 m, 2 m: n-2 m} \mathbf{P f}[X]_{2 m}$ & $\mathbf{P f}^{\prime} \Psi$ \\
EMf & $\mathbf{P f}^{\prime}[\Psi]_{n-2 m, 2 m ; n-2 m} \mathbf{P f}[\mathcal{X}]_{2 m}$ & $\mathbf{P f}^{\prime} \Psi$ \\
BI & $\left(\mathbf{P f}^{\prime} A\right)^{2}$ & $\mathbf{P f}^{\prime} \Psi$ \\
YMS & $\mathcal{C}_{\operatorname{Tr}_{1}} \cdots \mathcal{C}_{T_{m}} \sum_{\{i, j\}} \mathcal{P}_{\{i, j\}}(n, l, m)$ & $\mathcal{C}_{n}(\sigma)$ \\
SYMS & $\mathbf{P f}^{\prime}[\Psi]_{n-2 m, 2 m ; n-2 m} \mathbf{P f}[\mathcal{X}]_{2 m}$ & $\mathcal{C}_{n}(\sigma)$ \\
BAS & $\mathcal{C}_{n}(\widetilde{\sigma})$ & $\mathcal{C}_{n}(\sigma)$ \\
NLSM & $\left(\mathbf{P f}^{\prime} A\right)^{2}$ & $\mathcal{C}_{n}(\sigma)$ \\
$\phi^{4}$ & $\mathbf{P f}^{\prime} A \mathbf{P f}[X]_{n}$ & $\mathcal{C}_{n}(\sigma)$ \\
exDBI & $\mathcal{C}_{\operatorname{Tr}_{1}} \cdots \mathcal{C}_{T_{m}} \sum_{\{i, j\}} \mathcal{P}_{\{i, j\}}(n, l, m)$ & $\left(\mathbf{P f}^{\prime} A\right)^{2}$ \\
DBI & $\mathbf{P f}^{\prime}[\Psi]_{n-2 m, 2 m ; n-2 m} \mathbf{P f}[\mathcal{X}]_{2 m}$ & $\left(\mathbf{P f}^{\prime} A\right)^{2}$ \\
SG & $\left(\mathbf{P f}^{\prime} A\right)^{2}$ & $\left(\mathbf{P f}^{\prime} A\right)^{2}$ \\
\hline
\end{tabular}

and

$X_{i j}=\left\{\begin{array}{ll}\frac{1}{z_{i j}} & i \neq j, \\ 0 & i=j,\end{array} \quad \mathcal{X}_{i j}= \begin{cases}\frac{\delta^{I_{i}, I_{j}}}{z_{i j}} & i \neq j, \\ 0 & i=j .\end{cases}\right.$

The elements $\eta_{i j}$ are given as [29]

$\eta_{j \pm 1, j}=\frac{1}{2} \epsilon_{j} \cdot k_{j}$

$\eta_{i j}=0, \quad$ otherwise,

satisfy

$$
\sum_{i \neq j, i=1}^{n} \eta_{i j}=\epsilon_{j} \cdot k_{j}
$$

They render $C_{i i}$ to weight- 2 under the $\operatorname{SL}(2, \mathbb{C})$ transformation, and therefore keep the $\operatorname{SL}(2, \mathbb{C})$ invariance of the whole theory. $\delta^{I_{i}, I_{j}}$ is the Kronecker symbol, which forbids the interaction between particles with different flavors $I_{i}$ and $I_{j}$. When the dimension of a matrix needs to be made explicit, we often denote the $n \times n$ matrix $S$ as $[S]_{n}$. The $2 n \times 2 n$ antisymmetric matrix $\Psi$ can be constructed from the matrices $A$, $B$ and $C$, in the following form:

$\Psi=\left(\begin{array}{c|c}A & C \\ \hline-C^{\mathrm{T}} & B\end{array}\right)$

The reduced Pfaffian of $\Psi$ is defined as $\mathbf{P f}^{\prime} \Psi=\frac{(-)^{i+j}}{z_{i j}} \mathbf{P f} \Psi_{i j}^{i j}$, where the notation $\Psi_{i j}^{i j}$ means the $i$ th and $j$ th rows and columns in the matrix $\Psi$ have been removed (with $1 \leq i<$ $j \leq n)$. An analogous notation holds for $\mathbf{P f}^{\prime} A$.

It is worth to emphasize the definition of Pfaffian, since it is crucial for the work in this paper. For a $2 n \times 2 n$ antisymmetric matrix $S$, the Pfaffian is defined as 
$\operatorname{Pf} S=\frac{1}{2^{n} n !} \sum_{\sigma \in S_{2 n}} \operatorname{sgn}(\sigma) \prod_{i=1}^{n} S_{\sigma(2 i-1), \sigma(2 i)}$,

where $S_{2 n}$ denotes permutations of $2 n$ elements and $\operatorname{sgn}(\sigma)$ is the signature of $\sigma$. More explicitly, let $\Pi$ be the set of all partitions of $\{1,2, \ldots, 2 n\}$ into pairs without regarding the order. An element $\alpha$ in $\Pi$ can be written as

$\alpha=\left\{\left(i_{1}, j_{1}\right),\left(i_{2}, j_{2}\right), \ldots,\left(i_{n}, j_{n}\right)\right\}$,

with $i_{k}<j_{k}$ and $i_{1}<i_{2}<\cdots<i_{n}$. Now let

$\pi_{\alpha}=\left(\begin{array}{ccccccc}1 & 2 & 3 & 4 & \cdots & 2 n-1 & 2 n \\ i_{1} & j_{1} & i_{2} & j_{2} & \cdots & i_{n} & j_{n}\end{array}\right)$

be the corresponding permutation of the partition $\alpha$. If we define

$S_{\alpha}=\operatorname{sgn}\left(\pi_{\alpha}\right) S_{i_{1} j_{1}} S_{i_{2} j_{2}} \cdots S_{i_{n} j_{n}}$,

then the Pfaffian of the matrix $S$ is given as

$\operatorname{Pf} S=\sum_{\alpha \in \Pi} S_{\alpha}$.

From the definition (18), one can observe that, in every term $S_{\alpha}$ of the Pfaffian, each number in the set $\{1,2, \ldots, 2 n\}$, which serves as the subscript of the matrix element, will appear once and only once. This simple observation indicates that each polarization vector $\epsilon_{i}$ appears once and only once in each term of the reduced Pfaffian $\mathbf{P f}^{\prime} \Psi$. This conclusion is important for the later discussions.

The definition of $\Psi$ can be generalized to the $(2 a+b) \times$ $(2 a+b)$ matrix $[\Psi]_{a, b: a}$ as

$[\Psi]_{a, b: a}=\left(\begin{array}{c|c}A_{(a+b) \times(a+b)} & C_{(a+b) \times a} \\ \hline-C_{a \times(a+b)}^{\mathrm{T}} & B_{a \times a}\end{array}\right)$,

here $A$ is a $(a+b) \times(a+b)$ matrix, $C$ is a $(a+b) \times a$ matrix, and $B$ is a $a \times a$ matrix. The definitions of elements in $A, B$ and $C$ are the same as in (9). The reduced Pfaffian $\mathbf{P f}^{\prime}[\Psi]_{a, b: a}$ can be defined in the same manner.

Starting from the matrix $[\Psi]_{a, b: a}$, the polynomial $\mathcal{P}_{\{i, j\}}(n, l, m)$ is defined by

$$
\begin{aligned}
\mathcal{P}_{\{i, j\}}(n, l, m)= & \operatorname{sgn}(\{i, j\}) z_{i_{1} j_{1}} \cdots z_{i_{m} j_{m}} \\
& \operatorname{Pf}^{\prime}[\Psi]_{n-l, i_{1}, j_{1}, \ldots, i_{m}, j_{m}: n-l} \\
= & -\operatorname{sgn}\left(\{i, j\}^{\prime}\right) z_{i_{1} j_{1}} \cdots z_{i_{m-1} j_{m-1}} \\
& \mathbf{P f}[\Psi]_{n-l, i_{1}, j_{1}, \ldots, i_{m-1}, j_{m-1}: n-l},
\end{aligned}
$$

where $i_{k}<j_{k} \in \operatorname{Tr}_{k}$ and $\operatorname{Tr}_{k}$ are $m$ sets satisfying ${ }^{4}$

$\operatorname{Tr}_{1} \cup \operatorname{Tr}_{2} \cup \cdots \cup \operatorname{Tr}_{m}=\{n-l+1, n-l+2, \ldots, n\}$.

In the notation $[\Psi]_{n-l, i_{1}, j_{1}, \ldots, i_{m}, j_{m}: n-l}$, we explicitly write down $\left\{i_{1}, j_{1}, \ldots, i_{m}, j_{m}\right\}$ instead of $2 m$, to emphasize

\footnotetext{
${ }^{4}$ Each set has at least two elements, so in general we have $l \geq 2 m$.
}

the locations of these $2 m$ rows and $2 m$ columns in the original matrix $\Psi$. The two signatures $\operatorname{sgn}(\{i, j\})$ and $\operatorname{sgn}\left(\{i, j\}^{\prime}\right)$ correspond to partitions $\left\{\left(i_{1}, j_{1}\right), \ldots,\left(i_{m}, j_{m}\right)\right\}$ and $\left\{\left(i_{1}, j_{1}\right), \ldots,\left(i_{m-1}, j_{m-1}\right)\right\}$, respectively, and one can verify $\mathbf{s g n}(\{i, j\})=\mathbf{s g n}\left(\{i, j\}^{\prime}\right)$. In the second line of (20), the reduced Pfaffian is calculated by removing rows and columns $i_{m}$ and $j_{m}$. Based on the definition of $\mathcal{P}_{\{i, j\}}(n, l, m)$ in the second line of (20), the polynomial $\sum_{\{i, j\}}{ }^{\prime} \mathcal{P}_{\{i, j\}}(n, l, m)$ is defined as

$$
\sum_{\{i, j\}}^{\prime} \mathcal{P}_{\{i, j\}}(n, l, m) \equiv \sum_{\substack{i_{1}<j_{1} \in \operatorname{Tr}_{1} \\ i_{m-1}<j_{m-1} \in \operatorname{Tr}_{m-1}}} \mathcal{P}_{\{i, j\}}(n, l, m),
$$

where the sum is over all possible choices of pairs in each trace-subset $\operatorname{Tr}_{k}$. In the on-shell case, the polynomial $\sum_{\{i, j\}}{ }^{\prime} \mathcal{P}_{\{i, j\}}(n, l, m)$ defined above equals the reduced Pfaffian of the matrix $\Pi$, which is constructed from $\Psi$ via the squeezing procedure [5]. In this paper, we will not use the matrix $\Pi$. The advantage of this choice is that the polynomial $\sum_{\{i, j\}}{ }^{\prime} \mathcal{P}_{\{i, j\}}(n, l, m)$ defined in (22) is manifestly weight-2 under the $\operatorname{SL}(2, \mathbb{C})$ transformation, while the reduced Pfaffian of $\Pi$ does not have a manifest weight.

Finally, the Parke-Taylor factor for the ordering $\sigma$ is given by

$\mathcal{C}_{n}(\sigma)=\frac{1}{z_{\sigma_{1} \sigma_{2}} z_{\sigma_{2} \sigma_{3}} \cdots z_{\sigma_{n-1} \sigma_{n}} z_{\sigma_{n} \sigma_{1}}}$.

It indicates the color-ordering $\left(\sigma_{1}, \sigma_{2}, \ldots, \sigma_{n-1}, \sigma_{n}\right)$ among $n$ external legs.

With the ingredients introduced above, off-shell CHY integrands for various theories can be defined as in Table 1 . Some remarks are in order. First, the $\operatorname{SL}(2, \mathbb{C})$ symmetry plays the central role in the construction of off-shell CHY integrals. From on-shell integrals to off-shell ones, all corrections $\Delta_{i j}$ and $\eta_{i j}$ are required by the $\operatorname{SL}(2, \mathbb{C})$ invariance. It is straightforward to see that both $\mathcal{I}_{L}$ and $\mathcal{I}_{R}$ defined in Table 1 are weight- 2 under the $\operatorname{SL}(2, \mathbb{C})$ transformation, guarantee the $\operatorname{SL}(2, \mathbb{C})$ invariance of the whole integral. Secondly, all the reduced Pfaffians appear in off-shell CHY integrands in Table 1 are independent of the removed rows and columns, similar to those in on-shell integrands. One can follow the method used in [2], and use

$$
\sum_{i=1, i \neq j}^{n} k_{i} \cdot k_{j}+\Delta_{i j}=0, \quad \sum_{i=1, i \neq j}^{n} k_{i} \cdot \epsilon_{j}+\eta_{i j}=0,
$$

as well as scattering equations to prove this. Thirdly, the gauge invariance no longer exists in general. For example, under the replacement $\epsilon_{i} \rightarrow k_{i}$, we do not have $\operatorname{Pf}^{\prime} \Psi=0$ anymore, unless we take all $\Delta_{i j}$ and $\eta_{i j}$ to be 0 . But it is quite natural that the off-shell or massive external states violate gauge invariance. 


\subsection{Double-cover prescription}

In the following sections, all CHY integrals under consideration are in the double-cover formalism. In this subsection, we describe how to reformulate the single-cover $\mathrm{CHY}$ integral defined in (3) into the double-cover one.

The double-cover prescription of CHY construction is given as a contour integral on $n$-punctured double-covered Riemann spheres [22-27]. Restricted to the curves $0=$ $C_{i} \equiv y_{i}^{2}-\sigma_{i}^{2}+\Lambda^{2}$ for $i \in\{1,2, \ldots, n\}$, the pairs $\left(y_{1}, \sigma_{1}\right),\left(y_{2}, \sigma_{2}\right), \ldots,\left(y_{n}, \sigma_{n}\right)$ serve as coordinates. Then all $\frac{1}{z_{i j}}$ in the single-covered formula (3) are replaced by

$\tau_{i j} \equiv \frac{1}{2}\left(\frac{y_{i}+y_{j}+\sigma_{i j}}{y_{i}}\right) \frac{1}{\sigma_{i j}}$.

Especially, the scattering equations are turned to

$0=\mathcal{E}_{i}^{\tau} \equiv \sum_{j \in\{1,2, \ldots, n\} \backslash\{i\}}\left(2 k_{i} \cdot k_{j}+2 \Delta_{i j}\right) \tau_{i j}$.

The amplitudes in such a framework are expressed as the contour integral

$\mathcal{A}_{n}=\int d \mu_{n}^{\Lambda} \frac{\mathcal{I}_{L}^{\tau}\left(\sigma_{i}, y_{i}, k_{i}, \epsilon_{i}\right) \mathcal{I}_{R}^{\tau}\left(\sigma_{i}, y_{i}, k_{i}, \tilde{\epsilon}_{i}\right)}{\mathcal{E}_{m}^{\tau}}$,

where the measure $d \mu_{n}^{\Lambda}$ is defined through

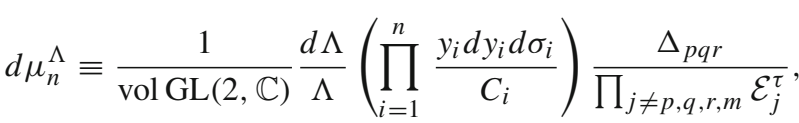

with $\Delta_{p q r} \equiv\left(\tau_{p q} \tau_{q r} \tau_{r p}\right)^{-1}$. Correspondingly, the contour is determined by the poles $\Lambda=0, C_{i}=0$, and by $\mathcal{E}_{j}^{\tau}=0$ holding for $j \neq p, q, r, m$. Eliminating the $\operatorname{GL}(2, \mathbb{C})$ gauge redundancy turns the measure to

$d \mu_{n}^{\Lambda} \equiv \frac{1}{2^{2}} \frac{d \Lambda}{\Lambda}\left(\prod_{i=1}^{n} \frac{y_{i} d y_{i}}{C_{i}}\right)\left(\prod_{j \neq p, q, r, m} \frac{d \sigma_{j}}{\mathcal{E}_{j}^{\tau}}\right) \Delta_{p q r} \Delta_{p q r \mid m}$,

where

$\Delta_{p q r \mid m} \equiv \sigma_{p} \Delta_{q r m}-\sigma_{q} \Delta_{r m p}+\sigma_{r} \Delta_{m p q}-\sigma_{m} \Delta_{p q r}$.

Then we consider $\mathcal{I}_{L}^{\tau}\left(\sigma_{i}, y_{i}, k_{i}, \epsilon_{i}\right)$ and $\mathcal{I}_{R}^{\tau}\left(\sigma_{i}, y_{i}, k_{i}, \widetilde{\epsilon}_{i}\right)$, which are obtained from $\mathcal{I}_{L}\left(z_{i}, k_{i}, \epsilon_{i}\right)$ and $\mathcal{I}_{R}\left(z_{i}, k_{i}, \widetilde{\epsilon}_{i}\right)$ in Table 1 via the replacement $\frac{1}{z_{i j}} \rightarrow \tau_{i j}$. To obtain the doublecover form of the integrand, which is more convenient for our consideration, we rewrite $\tau_{i j}$ as

$\tau_{i j}=\frac{(y \sigma)_{i}}{y_{i}} T_{i j} \equiv \frac{(y \sigma)_{i}}{y_{i}} \frac{1}{(y \sigma)_{i}-(y \sigma)_{j}}$, on the support $C_{i}=C_{j}=0$. The advantage of this reformulation is that $T_{i j}$ has algebraic properties similar to $\frac{1}{z_{i j}}$, such as antisymmetry, and

$T_{i k}-T_{j k}=\frac{T_{i k} T_{j k}}{T_{j i}}$.

This similarity allows us to use a lot of technics for singlecover CHY integrals. Under the replacement (31), we have ${ }^{5}$

$$
\begin{aligned}
& \mathcal{I}_{L}^{\tau}\left(\sigma_{i}, y_{i}, k_{i}, \epsilon_{i}\right)=\left(\prod_{i=1}^{n} \frac{(y \sigma)_{i}}{y_{i}}\right) \mathcal{I}_{L}^{\Lambda}\left(T_{i j}, k_{i}, \epsilon_{i}\right), \\
& \mathcal{I}_{R}^{\tau}\left(\sigma_{i}, y_{i}, k_{i}, \widetilde{\epsilon}_{i}\right)=\left(\prod_{i=1}^{n} \frac{(y \sigma)_{i}}{y_{i}}\right) \mathcal{I}_{R}^{\Lambda}\left(T_{i j}, k_{i}, \widetilde{\epsilon}_{i}\right) .
\end{aligned}
$$

For example, the Parke-Taylor factor becomes

$$
\begin{aligned}
\mathcal{C}_{n}^{\tau}(\sigma) & =\left(\prod_{i=1}^{n} \frac{(y \sigma)_{i}}{y_{i}}\right) \mathcal{C}_{n}^{\Lambda}(\sigma) \\
& \equiv\left(\prod_{i=1}^{n} \frac{(y \sigma)_{i}}{y_{i}}\right) T_{\sigma_{1} \sigma_{2}} T_{\sigma_{2} \sigma_{3}} \cdots T_{\sigma_{n} \sigma_{1}}
\end{aligned}
$$

Thus, starting from an integrand in the single-cover version, one can first replace all $\frac{1}{z_{i j}}$ by $T_{i j}$, and then multiply the resulting formula by the factor $\left(\prod_{i=1}^{n} \frac{(y \sigma)_{i}}{y_{i}}\right)$. Let us take $\mathbf{P f ^ { \prime }} \Psi$ as the example, the reduced Pfaffian with new coordinates $y_{i}$ and $\sigma_{i}$ is given by

$\mathbf{P f}^{\prime} \Psi^{\tau} \equiv\left(\prod_{i=1}^{n} \frac{(y \sigma)_{i}}{y_{i}}\right)(-)^{a+b} T_{a b} \mathbf{P f}\left(\Psi^{\Lambda}\right)_{a b}^{a b}$,

where the matrix $\Psi^{\Lambda}$ is obtained from $\Psi$ via the replacement $\frac{1}{z_{i j}} \rightarrow T_{i j}$.

\section{Effects of basic operators}

As discussed in Sect. 1, we are interested in acting differential operators on off-shell CHY integrals in the double-cover version. To achieve this goal, it is sufficient to apply the differential operators to $\mathcal{I}_{L}^{\Lambda}\left(T_{i j}, k_{i}, \epsilon_{i}\right)$ and $\mathcal{I}_{R}^{\Lambda}\left(T_{i j}, k_{i}, \widetilde{\epsilon}_{i}\right)$, since the operators under consideration will not affect both the measure and the factor $\frac{(y \sigma)_{i}}{y_{i}}$. In this section, we will consider the effects of applying three basic differential operators to the elementary building blocks of $\mathcal{I}_{L}^{\Lambda}\left(T_{i j}, k_{i}, \epsilon_{i}\right)$ and $\mathcal{I}_{R}^{\Lambda}\left(T_{i j}, k_{i}, \tilde{\epsilon}_{i}\right)$, such as $\mathbf{P f}^{\prime} \Psi^{\Lambda}, \mathbf{P f}^{\prime}[\Psi]_{a, b: a}^{\Lambda}$ and $\sum_{\{i, j\}}^{\prime}$ $\mathcal{P}_{\{i, j\}}^{\Lambda}(n, l, m)$. Among the operators which will be discussed in this section, the trace and insertion operators are the same

\footnotetext{
$\overline{5}$ Maybe the notations $\mathcal{I}_{L}^{T}\left(T_{i j}, k_{i}, \epsilon_{i}\right)$ and $\mathcal{I}_{R}^{T}\left(T_{i j}, k_{i}, \widetilde{\epsilon}_{i}\right)$ are more suitable. However, when encountering matrices, notations such as $\Psi^{T}, A^{T}$ will cause some ambiguity, since we always use $T$ to denote the transpose of the matrix. Thus we choose the superscript to be $\Lambda$ rather than $T$.
} 
as those defined in [6], while the definition of the longitudinal operators will be modified.

\subsection{Trace operator}

The trace operator $\mathcal{T}[i, j]$ is defined as [6]

$\mathcal{T}[i, j] \equiv \frac{\partial}{\partial\left(\epsilon_{i} \cdot \epsilon_{j}\right)}$.

If one applies $\mathcal{T}[i, j]$ to the reduced Pfaffian $\mathbf{P f}^{\prime} \Psi^{\Lambda}$, only terms containing the factor $\left(\epsilon_{i} \cdot \epsilon_{j}\right)$ (i.e., the elements $\Psi_{i+n, j+n}^{\Lambda}$ ) provide non-vanishing contributions. Since $\epsilon_{i}$ and $\epsilon_{j}$ appear once and only once in each term of the reduced Pfaffian, performing the operator $\mathcal{T}[i, j]$ is equivalent to the replacement

$\epsilon_{i} \cdot \epsilon_{j} \rightarrow 1, \quad \epsilon_{i} \cdot V \rightarrow 0, \quad \epsilon_{j} \cdot V \rightarrow 0$,

where $V$ denotes vectors $k_{l}$ or $\epsilon_{l \neq i, j}$. This manipulation is equivalent to the dimensional reduction procedure in [5]. Then we arrive at a new matrix $\widetilde{\Psi}^{\Lambda}$, satisfying

$\mathcal{T}_{i j} \mathbf{P f}^{\prime} \Psi^{\Lambda}=\mathbf{P f}^{\prime} \widetilde{\Psi}^{\Lambda}$.

Without loss of generality, we may assume $\{i, j\}=\{n-$ $1, n\},{ }^{6}$ then the new matrix $\widetilde{\Psi}^{\Lambda}$ is given by

$$
\begin{aligned}
\widetilde{\Psi}^{\Lambda} & =\left(\begin{array}{c|c|c}
A_{n \times n}^{\Lambda} & C_{n \times(n-2)}^{\Lambda} & 0 \\
\hline-\left(C^{\Lambda}\right)_{(n-2) \times n}^{\mathrm{T}} & B_{(n-2) \times(n-2)}^{\Lambda} & 0 \\
\hline 0 & 0 & X_{2 \times 2}^{\Lambda}
\end{array}\right) \\
& =\left(\begin{array}{c|c}
{[\Psi]_{n-2,2: n-2}^{\Lambda}} & 0 \\
\hline 0 & {[X]_{2}^{\Lambda}}
\end{array}\right),
\end{aligned}
$$

where the matrices $[\Psi]_{n-2,2: n-2}^{\Lambda}$ and $[X]_{2}^{\Lambda}$ are obtained from $[\Psi]_{n-2,2: n-2}$ and $[X]_{2}$ through the replacement $\frac{1}{z_{i j}} \rightarrow T_{i j}$. The reduced Pfaffian of the matrix $\widetilde{\Psi}^{\Lambda}$ can be calculated straightforwardly to be

$\mathbf{P f}^{\prime} \widetilde{\Psi}^{\Lambda}=\mathbf{P f}^{\prime}[\Psi]_{n-2,2 ; n-2}^{\Lambda} \mathbf{P f}[X]_{2}^{\Lambda}$.

Thus, we find

$\mathcal{T}[i, j] \mathbf{P f}^{\prime} \Psi^{\Lambda}=\mathbf{P f}^{\prime}[\Psi]_{n-2,2 ; n-2}^{\Lambda} \mathbf{P f}[X]_{2}^{\Lambda}$.

Applying a similar procedure to the matrix $[\Psi]_{n-2,2: n-2}^{\Lambda}$ gives

$\mathcal{T}[i, j] \mathbf{P f}^{\prime}[\Psi]_{n-2,2: n-2}^{\Lambda}=\mathbf{P f}^{\prime}[\Psi]_{a-4,4: a-4}^{\Lambda} \mathbf{P f}[X]_{2}^{\Lambda}$.

By repeating the manipulation, we find that the multiple action of trace operators gives the following recursive pattern:

6 This assumption can be realized by moving lows and columns. Since the $(n+i)$ th row and column will be moved simultaneously while moving the $i$ th ones, the possible minus sign will be canceled.

$$
\begin{aligned}
\mathcal{T} & {\left[i_{1}, j_{1}\right] \mathcal{T}\left[i_{2}, j_{2}\right] \mathbf{P f}^{\prime} \Psi^{\Lambda} } \\
= & \mathbf{P f}^{\prime}[\Psi]_{n-4,4 ; n-4}^{\Lambda} \mathbf{P f}\left[X_{1}\right]_{2}^{\Lambda} \mathbf{P f}\left[X_{2}\right]_{2}^{\Lambda}, \\
& \ldots \\
\mathcal{T} & {\left[i_{1}, j_{1}\right] \mathcal{T}\left[i_{2}, j_{2}\right] \cdots \mathcal{T}\left[i_{m}, j_{m}\right] \mathbf{P f}^{\prime} \Psi^{\Lambda} } \\
= & \mathbf{P f}^{\prime}[\Psi]_{n-2 m, 2 m ; n-2 m}^{\Lambda} \mathbf{P f}\left[X_{1}\right]_{2}^{\Lambda} \mathbf{P f}\left[X_{2}\right]_{2}^{\Lambda} \cdots \mathbf{P f}\left[X_{m}\right]_{2}^{\Lambda} \\
= & (-)^{m}\left(T_{i_{1} j_{1}} T_{j_{1} i_{1}}\right)\left(T_{i_{2} j_{2}} T_{j_{2} i_{2}}\right) \cdots\left(T_{i_{m} j_{m}} T_{j_{m} i_{m}}\right) \\
& \times \mathcal{P}_{\{i, j\}}^{\Lambda}(n, 2 m, m),
\end{aligned}
$$

where the polynomial $\mathcal{P}_{\{i, j\}}^{\Lambda}(n, l, m)$ is obtained from $\mathcal{P}_{\{i, j\}}(n, l, m)$ in (20) via the replacement $\frac{1}{z_{i j}} \rightarrow T_{i j}$, and we have arranged elements as

$\left[X_{k}\right]_{2}^{\Lambda}=\left(\begin{array}{c|c}0 & T_{i_{k} j_{k}} \\ \hline T_{j_{k} i_{k}} & 0\end{array}\right)$.

We want to point out that the factor $\left(T_{i j} T_{j i}\right)$ appearing in (43) is the simplest Parke-Taylor factor $\mathcal{C}_{2}^{\Lambda}$ defined in (34), which indicates the simplest color-ordering $(i, j)$. More general color-orderings can be generated from it by inserting other elements.

\subsection{Insertion operator}

The insertion operator is defined by [6]

$\mathcal{I}_{i k j} \equiv \frac{\partial}{\partial\left(k_{i} \cdot \epsilon_{k}\right)}-\frac{\partial}{\partial\left(k_{j} \cdot \epsilon_{k}\right)}$.

In this subsection, we discuss the effect of acting this operator on the polynomial $\sum_{\{i, j\}} \mathcal{P}_{\{i, j\}}^{\Lambda}(n, l, m)$. As will be seen immediately, the most important effect is replacing $T_{i j}$ in the Parke-Taylor factor by $T_{i k} T_{k j}$. In other words, this operator transmutes the color-ordering $(\ldots, i, j, \ldots)$ to $(\ldots, i, k, j, \ldots)$. To show this, one needs to assume that the $i$ th and $j$ th legs belong to the same trace subset, i.e., $i, j \in \operatorname{Tr}_{k}$. For simplicity, we assume $i, j \in \operatorname{Tr}_{m}$, and we take the expansion (20) where rows and columns $i_{m}, j_{m} \in \operatorname{Tr}_{m}$ have been removed (with replacing $\frac{1}{z_{i j}}$ by $T_{i j}$ ). Since in the off-shell case the reduced Pfaffian is also independent of the choice of removed rows and columns, as pointed out in the previous section, assuming $i$ and $j$ belong to any other $\operatorname{Tr}_{k}$ will not change the conclusion, although the calculation will be more complicated.

Let us consider the polynomial $\mathbf{P f}[\Psi]_{n-l, i_{1}, j_{1}, \ldots, i_{m-1}, j_{m-1}: n-l}^{\Lambda}$, which is given as

$$
\begin{aligned}
& \operatorname{Pf}[\Psi]_{n-l, i_{1}, j_{1}, \ldots, i_{m-1}, j_{m-1}: n-l}^{\Lambda} \\
& \quad=\sum_{\alpha \in \Pi} \operatorname{sgn}\left(\pi_{\alpha}\right)[\Psi]_{a_{1} b_{1}}^{\Lambda}[\Psi]_{a_{2} b_{2}}^{\Lambda} \cdots[\Psi]_{a_{\left(n^{\prime}+m^{\prime}\right)} b_{\left(n^{\prime}+m^{\prime}\right)}}^{\Lambda},
\end{aligned}
$$

where the definition of Pfaffian in (18) has been used. The element $[\Psi]_{a_{i} b_{i}}^{\Lambda}$ is at the $a_{i}$ th row and $b_{i}$ th column in the matrix $[\Psi]_{n-l, i_{1}, j_{1}, \ldots, i_{m-1}, j_{m-1}: n-l}^{\Lambda}$, and we have defined $n^{\prime}=n-l$, 
$m^{\prime}=m-1$. Since we have chosen $i, j \in \operatorname{Tr}_{m}$, it is straightforward to see that $k_{i} \cdot \epsilon_{k}$ appears only in $C_{k k}^{\Lambda}$. Thus, when applying $\frac{\partial}{\partial\left(k_{i} \epsilon_{k}\right)}$ to (46), only terms containing the element $[\Psi]_{k, n^{\prime}+2 m^{\prime}+k}^{\Lambda}$ (see the formula (19)) can survive. For such a term, the remaining part after eliminating $[\Psi]_{k, n^{\prime}+2 m^{\prime}+k}^{\Lambda}$ corresponds to a partition of the set $\left\{1,2, \ldots, 2\left(n^{\prime}+m^{\prime}\right)\right\} \backslash$ $\left\{k, n^{\prime}+2 m^{\prime}+k\right\}$, which has the length $2\left(n^{\prime}+m^{\prime}-1\right)$. Such a term appears in the $\mathbf{P f}[\Psi]_{n-l-1, i_{1}, j_{1}, \ldots, i_{m-1}, j_{m-1}: n-l-1}^{\Lambda}$, weighted by a new signature $\operatorname{sgn}(\pi \widetilde{\alpha})$, where the new matrix $[\Psi]_{n-l-1, i_{1}, j_{1}, \ldots, i_{m-1}, j_{m-1}: n-l-1}^{\Lambda}$ is obtained from the original one $[\Psi]_{n-l, i_{1}, j_{1}, \ldots, i_{m-1}, j_{m-1}: n-l}^{\Lambda}$ by removing the $k$ th and $\left(n^{\prime}+2 m^{\prime}+k\right)$ th rows and columns, and $\mathbf{s g n}(\pi \widetilde{\alpha})$ corresponds to the partition of the length-2(n'+ $\left.m^{\prime}-1\right)$ set. Comparing two special partitions, where one corresponds to the original matrix, the other one corresponds to the new matrix,

$$
\begin{aligned}
& \alpha=\left\{\left(a_{1}, b_{1}\right),\left(a_{2}, b_{2}\right), \ldots,\left(k, n^{\prime}+2 m^{\prime}+k\right),\right. \\
&\left.\ldots,\left(a_{\left(n^{\prime}+m^{\prime}\right)}, b_{\left(n^{\prime}+m^{\prime}\right)}\right)\right\}, \\
& \widetilde{\alpha}=\left\{\left(a_{1}, b_{1}\right),\left(a_{2}, b_{2}\right), \ldots,\left(a_{\left(n^{\prime}+m^{\prime}-1\right)}, b_{\left(n^{\prime}+m^{\prime}-1\right)}\right)\right\},
\end{aligned}
$$

one can find $\operatorname{sgn}\left(\pi_{\alpha}\right)=(-)^{n^{\prime}-1} \operatorname{sgn}\left(\pi_{\widetilde{\alpha}}\right)$. Thus, summing all contributions gives

$$
\begin{aligned}
& \frac{\partial}{\partial\left(k_{i} \cdot \epsilon_{k}\right)} \mathbf{P f}[\Psi]_{n-l, i_{1}, j_{1}, \ldots, i_{m-1}, j_{m-1}: n-l}^{\Lambda} \\
& \quad=(-)^{n-l} T_{i k} \mathbf{P f}[\Psi]_{n-l-1, i_{1}, j_{1}, \ldots, i_{m-1}, j_{m-1}: n-l-1}^{\Lambda} .
\end{aligned}
$$

Applying the above result to (20), we get

$$
\begin{aligned}
& \mathcal{T}_{i k j}\left(\sum_{\{i, j\}}^{\prime} \mathcal{P}_{\{i, j\}}^{\Lambda}(n, l, m)\right) \\
& =(-)^{n-l}\left(T_{i k}-T_{j k}\right)\left(\sum_{\{i, j\}}^{\prime} \mathcal{P}_{\{i, j\}}^{\Lambda}(n, l+1, m)\right) \\
& =(-)^{n-l} \frac{T_{i k} T_{k j}}{T_{i j}}\left(\sum_{\{i, j\}}^{\prime} \mathcal{P}_{\{i, j\}}^{\Lambda}(n, l+1, m)\right),
\end{aligned}
$$

where the definition

$$
T_{i j} \equiv \frac{1}{(y \sigma)_{i}-(y \sigma)_{j}}
$$

has been used. The factor $\frac{T_{i k} T_{k j}}{T_{i j}}$ in the result (49) indicates that the insertion operator transmutes $T_{i j}$ in the Parke-Taylor factor to $T_{i k} T_{k j}$; thus it inserts the $k$ th external leg between $i$ th and $j$ th legs in the color-ordering.

\subsection{Longitudinal operator}

For on-shell amplitudes, the longitudinal operators are defined via [6]

$\mathcal{L}_{i}^{\prime} \equiv \sum_{j \neq i}\left(k_{i} \cdot k_{j}\right) \frac{\partial}{\partial\left(k_{j} \cdot \epsilon_{i}\right)}$

and

$\mathcal{L}_{i j}^{\prime} \equiv-\left(k_{i} \cdot k_{j}\right) \frac{\partial}{\partial\left(\epsilon_{i} \cdot \epsilon_{j}\right)}$.

For the off-shell case, they should be modified to

$\mathcal{L}_{i} \equiv \sum_{j \neq i}\left(k_{i} \cdot k_{j}+\Delta_{i j}\right) \frac{\partial}{\partial\left(k_{j} \cdot \epsilon_{i}\right)}$

and

$\mathcal{L}_{i j} \equiv-\left(k_{i} \cdot k_{j}+\Delta_{i j}\right) \frac{\partial}{\partial\left(\epsilon_{i} \cdot \epsilon_{j}\right)}$.

The reason for the above modifications will be seen in the next section. We now discuss the effects of acting them on the reduced Pfaffian $\mathbf{P f}^{\prime}[\Psi]_{a, b: a}^{\Lambda}$.

We first consider the operator $\mathcal{L}_{i j}$. It turns $\left(\epsilon_{i} \cdot \epsilon_{j}\right)$ to $\left(k_{i} \cdot k_{j}+\Delta_{i j}\right)$, and annihilates all other $\left(\epsilon_{i} \cdot V\right),\left(\epsilon_{j} \cdot V\right)$. Using the observation that $\epsilon_{i}$ and $\epsilon_{j}$ appear once and only once, respectively, one can conclude that $\mathcal{L}_{i j}$ transmutes the reduced Pfaffian of the matrix $[\Psi]_{a, b: a}^{\Lambda}$ as follows:

$$
\begin{aligned}
\mathcal{L}_{i j} \mathbf{P f}^{\prime}\left(\begin{array}{c|c}
A_{(a+b) \times(a+b)}^{\Lambda} & C_{(a+b) \times a}^{\Lambda} \\
\hline-\left(C^{\Lambda}\right)_{a \times(a+b)}^{\mathrm{T}} & B_{a \times a}^{\Lambda}
\end{array}\right) \\
\Rightarrow \mathbf{P f}^{\prime}\left(\begin{array}{c|c|c}
A_{(a+b) \times(a+b)}^{\Lambda} & C_{(a+b) \times(a-2)}^{\Lambda} & 0 \\
\hline-\left(C^{\Lambda}\right)_{(a-2) \times(a+b)}^{\mathrm{T}} & B_{(a-2) \times(a-2)}^{\Lambda} & 0 \\
\hline 0 & 0 & -A_{2 \times 2}^{\Lambda}
\end{array}\right) .
\end{aligned}
$$

Then we consider the operator $\mathcal{L}_{i}$. It is straightforward to see

$\frac{\partial \eta_{j i}}{\partial\left(k_{j} \cdot \epsilon_{i}\right)}=0$,

thus the operator $\mathcal{L}_{i}$ transmutes every $\left(k_{j} \cdot \epsilon_{i}+\eta_{j i}\right)$ to $\left(k_{j}\right.$. $\left.k_{i}+\Delta_{i j}\right)$. Under such a replacement, the diagonal elements of the matrix $C^{\Lambda}$ are transmuted to

$C_{i i}^{\Lambda} \rightarrow-\sum_{l=1, l \neq i}^{n}\left(k_{l} \cdot k_{i}+\Delta_{l j}\right) T_{l i}$,

vanishing due to the scattering equations

$$
\begin{aligned}
0 & =\mathcal{E}_{i}^{\tau} \equiv \sum_{j=1, j \neq i}^{n}\left(2 k_{i} \cdot k_{j}+2 \Delta_{i j}\right) \tau_{i j} \\
& =\frac{(y \sigma)_{i}}{y_{i}} \sum_{j=1, j \neq i}^{n}\left(2 k_{i} \cdot k_{j}+2 \Delta_{i j}\right) T_{i j} .
\end{aligned}
$$


Thus, the operator $\mathcal{L}_{i}$ has the following effect:

$$
\begin{aligned}
& \mathcal{L}_{i} \mathbf{P f}^{\prime}\left(\begin{array}{c|c|c}
A_{(a+b) \times(a+b)}^{\Lambda} & C_{(a+b) \times a}^{\Lambda} \\
\hline-\left(C^{\Lambda}\right)_{a \times(a+b)}^{\mathrm{T}} & B_{a \times a}^{\Lambda}
\end{array}\right) \\
& \Rightarrow \mathbf{P f}^{\prime}\left(\begin{array}{c|c|c}
A_{(a+b) \times(a+b)}^{\Lambda} & C_{(a+b) \times(a-2)}^{\Lambda} & A_{(a+b) \times 2}^{\Lambda} \\
\hline-\left(C^{\Lambda}\right)_{(a-2) \times(a+b)}^{\mathrm{T}} & B_{(a-2) \times(a-2)}^{\Lambda} & 0 \\
\hline A_{2 \times(a+b)}^{\Lambda} & 0 & 0
\end{array}\right) .
\end{aligned}
$$

The results (55) and (59) are crucial for generating the ingredient $\left(\mathbf{P f}^{\prime} A\right)^{2}$.

\section{Effects of combinatory operators}

The combinatory operators are constructed by three types of basic operators. In this section, by using the results obtained in the previous section, we will consider three kinds of combinatory operators, especially their action on the reduced Pfaffian $\mathbf{P f}^{\prime} \Psi^{\Lambda}$, which is the fundamental building block for the GR integrand.

\subsection{Operator $\mathcal{T}[\alpha]$}

The general trace operator $\mathcal{T}[\alpha]$ for an ordered length- $m$ set $\alpha=\left\{\alpha_{1}, \alpha_{2}, \ldots, \alpha_{m}\right\}$ is defined as ${ }^{7}$ [6]

$\mathcal{T}[\alpha] \equiv \mathcal{T}\left[\alpha_{1}, \alpha_{m}\right] \cdot \prod_{i=2}^{m-1} \mathcal{I}_{\alpha_{i-1} \alpha_{i} \alpha_{m}}$

As discussed before, when acting on $\operatorname{Pf}^{\prime} \Psi^{\Lambda}$, the operator $\mathcal{T}\left[\alpha_{1}, \alpha_{m}\right]$ creates the Parke-Taylor factor $T_{\alpha_{1} \alpha_{m}} T_{\alpha_{m} \alpha_{1}}$. On the other hand, the insertion operator $\mathcal{I}_{\alpha_{i-1} \alpha_{i} \alpha_{m}}$ transmutes $T_{\alpha_{i-1} \alpha_{m}}$ to $T_{\alpha_{i-1} \alpha_{i}} T_{\alpha_{i} \alpha_{m}}$. Thus, we can expect that the operator $\mathcal{T}[\alpha]$ will create the Parke-Taylor factor $T_{\alpha_{1} \alpha_{2}} \cdots T_{\alpha_{m-1} \alpha_{m}} T_{\alpha_{m} \alpha_{1}}$, which indicates the color-ordering $\left(\alpha_{1}, \alpha_{2}, \ldots, \alpha_{m}\right)$.

To verify this expectation, let us compute the result of applying the operator $\mathcal{T}[\alpha]$ to $\mathbf{P f}^{\prime} \Psi^{\Lambda}$. At the first step, performing $\mathcal{T}\left[\alpha_{1}, \alpha_{m}\right]$ gives

$$
\begin{aligned}
\mathcal{T}\left[\alpha_{1}, \alpha_{m}\right] \mathbf{P f}^{\prime} \Psi^{\Lambda} & =T_{\alpha_{1} \alpha_{m}} \mathbf{P f}^{\prime}[\Psi]_{n-2, \alpha_{1}, \alpha_{m}: n-2}^{\Lambda} \\
& =T_{\alpha_{1} \alpha_{m}} T_{\alpha_{m} \alpha_{1}} \mathbf{P f}[\Psi]_{n-2: n-2}^{\Lambda},
\end{aligned}
$$

where the result (41) has been used. Then one can act $\mathcal{I}_{\alpha_{1} \alpha_{2} \alpha_{m}}$ on the resulting object, and use (49) to get

$$
\begin{aligned}
& \mathcal{I}_{\alpha_{1} \alpha_{2} \alpha_{m}} \mathcal{T}\left[\alpha_{1}, \alpha_{m}\right] \mathbf{P f}^{\prime} \Psi^{\Lambda} \\
& \quad=(-)^{n-2} T_{\alpha_{1} \alpha_{m}} T_{\alpha_{m} \alpha_{1}} \frac{T_{\alpha_{1} \alpha_{2}} T_{\alpha_{2} \alpha_{m}}}{T_{\alpha_{1} \alpha_{m}}} \operatorname{Pf}[\Psi]_{n-3: n-3}^{\Lambda}
\end{aligned}
$$

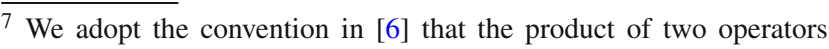
$\mathcal{O}_{1} \cdot \mathcal{O}_{2}$ acts on an amplitude as $\left(\mathcal{O}_{1} \cdot \mathcal{O}_{2}\right) \mathcal{A}=\mathcal{O}_{2} \mathcal{O}_{1} \mathcal{A}$, i.e., the operator $\mathcal{O}_{1}$ is performed at first, and $\mathcal{O}_{2}$ secondly.
}

$$
=(-)^{n-2} T_{\alpha_{1} \alpha_{2}} T_{\alpha_{2} \alpha_{m}} T_{\alpha_{m} \alpha_{1}} \mathbf{P f}[\Psi]_{n-3: n-3}^{\Lambda} .
$$

A similar manipulation gives

$$
\begin{aligned}
& \mathcal{I}_{\alpha_{2} \alpha_{3} \alpha_{m}} \mathcal{I}_{\alpha_{1} \alpha_{2} \alpha_{m}} \mathcal{T}\left[\alpha_{1}, \alpha_{m}\right] \mathbf{P f}^{\prime} \Psi^{\Lambda} \\
& =(-)^{n-2}(-)^{n-3} \frac{T_{\alpha_{1} \alpha_{2}} T_{\alpha_{2} \alpha_{m}} T_{\alpha_{m} \alpha_{1}} T_{\alpha_{2} \alpha_{3}} T_{\alpha_{3} \alpha_{m}}}{T_{\alpha_{2} \alpha_{m}}} \\
& \quad \times \operatorname{Pf}[\Psi]_{n-4: n-4}^{\Lambda} \\
& =(-)^{(n-2)+(n-3)} T_{\alpha_{1} \alpha_{2}} T_{\alpha_{2} \alpha_{3}} T_{\alpha_{3} \alpha_{m}} T_{\alpha_{m} \alpha_{1}} \\
& \quad \times \operatorname{Pf}[\Psi]_{n-4: n-4}^{\Lambda},
\end{aligned}
$$

and the recursive pattern can be observed. Repeating the above procedure, one will arrive at

$$
\begin{aligned}
\mathcal{T}[\alpha] \mathbf{P f}^{\prime} \Psi^{\Lambda}= & (-)^{\frac{(2 n-m-1)(m-2)}{2}} \\
& \times T_{\alpha_{1} \alpha_{2}} T_{\alpha_{2} \alpha_{3}} \cdots T_{\alpha_{m-1} \alpha_{m}} T_{\alpha_{m} \alpha_{1}} \mathbf{P f}[\Psi]_{n-m: n-m}^{\Lambda} \\
= & (-)^{\frac{(2 n-m-1)(m-2)}{2}+1} \mathcal{C}_{\alpha}^{\Lambda} \sum_{\{i, j\}}^{\prime} \mathcal{P}_{\{i, j\}}^{\Lambda}(n, m, 1) .
\end{aligned}
$$

Especially, when $m=n$, we have

$$
\begin{aligned}
\mathcal{T} & {\left[\alpha_{1}, \alpha_{2}, \ldots, \alpha_{n}\right] \mathbf{P f}^{\prime} \Psi^{\Lambda}=(-)^{\frac{(n-1)(n-2)}{2}} } \\
& T_{\alpha_{1} \alpha_{2}} T_{\alpha_{2} \alpha_{3}} \cdots T_{\alpha_{n-1} \alpha_{n}} T_{\alpha_{n} \alpha_{1}}=(-)^{\frac{(n-1)(n-2)}{2}} \mathcal{C}_{n}^{\Lambda} .
\end{aligned}
$$

The procedure of generating the color-ordering $\left(\alpha_{1}, \ldots, \alpha_{m}\right)$ can be understood as follows. At the first step, two reference points $\alpha_{1}$ and $\alpha_{m}$ are created by the operator $\mathcal{T}\left[\alpha_{1}, \alpha_{m}\right]$. Then the other legs in the set $\alpha$ are inserted between $\alpha_{1}$ and $\alpha_{m}$, via the insertion operators. This interpretation allows the trace operator $\mathcal{T}[\alpha]$ to have a variety of equivalent expressions, since different legs can be inserted by a variety of equivalent ways. As an example, let us consider the color-ordering $(1,2,3,4,5)$. To generate it, the first step can be creating two reference points 1 and 5 via the trace operator $\mathcal{T}[1,5]$. Then one can insert the other legs between 1 and 5 in the following order:

- inserts 2 between 1 and 5 ,

- inserts 3 between 2 and 5 ,

- inserts 4 between 3 and 5 .

This order yields the operator

$\mathcal{T}[1,2,3,4,5]=\mathcal{T}[1,5] \cdot \mathcal{I}_{125} \cdot \mathcal{I}_{235} \cdot \mathcal{I}_{345}$,

coinciding with the initial definition (60). However, one can also choose other equivalent orders, for instance:

- inserts 3 between 1 and 5 ,

- inserts 2 between 1 and 3 ,

- inserts 4 between 3 and 5 , 
and arrive at the operator

$\mathcal{T}^{\prime}[1,2,3,4,5]=\mathcal{T}[1,5] \cdot \mathcal{I}_{135} \cdot \mathcal{I}_{123} \cdot \mathcal{I}_{345}$

When applying to $\mathbf{P f}^{\prime} \Psi^{\Lambda}$, the two operators $\mathcal{T}[1,2,3,4,5]$ and $\mathcal{T}^{\prime}[1,2,3,4,5]$ are equivalent, as can be verified by the techniques from (61) to (64).

Furthermore, based on the cyclic symmetry of the colorordering, one can choose two arbitrary points $\alpha_{a}$ and $\alpha_{b}$ as reference points, then insert $\alpha_{a+1}, \ldots, \alpha_{b-1}$ between $\alpha_{a}$ and $\alpha_{b}$, and insert $\alpha_{b+1}, \ldots, \alpha_{a-1}$ between $\alpha_{b}$ and $\alpha_{a}$. For example, one can also choose the trace operator to be

$$
\begin{aligned}
\mathcal{T}[\alpha] \equiv & \mathcal{T}\left[\alpha_{2}, \alpha_{m-1}\right] \cdot \mathcal{I}_{\alpha_{m-1} \alpha_{m} \alpha_{2}} \cdot \mathcal{I}_{\alpha_{m} \alpha_{1} \alpha_{2}} \\
& \left(\prod_{i=3}^{m-2} \mathcal{I}_{\alpha_{i-1} \alpha_{i} \alpha_{m-1}}\right) .
\end{aligned}
$$

The previous result in the current subsection can be generalized to multi-trace cases $\mathcal{T}\left[\alpha_{1}\right] \cdot \mathcal{T}\left[\alpha_{2}\right] \cdots$, with the constraint $\left[\alpha_{i}\right] \cap\left[\alpha_{j}\right]=\emptyset$ for arbitrary $i$ and $j$. Let us consider, for example,

$$
\begin{aligned}
\mathcal{T}[\alpha] \cdot \mathcal{T}[\beta]= & \left(\mathcal{T}\left[\alpha_{1}, \alpha_{m}\right] \cdot \prod_{i=2}^{m-1} \mathcal{I}_{\alpha_{i-1} \alpha_{i} \alpha_{m}}\right) \\
& \cdot\left(\mathcal{T}\left[\beta_{1}, \beta_{l}\right] \cdot \prod_{i=2}^{l-1} \mathcal{I}_{\beta_{i-1} \beta_{i} \beta_{l}}\right) \\
= & \mathcal{T}\left[\alpha_{1}, \alpha_{m}\right] \cdot \mathcal{T}\left[\beta_{1}, \beta_{l}\right] \cdot\left(\prod_{i=2}^{m-1} \mathcal{I}_{\alpha_{i-1} \alpha_{i} \alpha_{m}}\right) \\
& \cdot\left(\prod_{i=2}^{l-1} \mathcal{I}_{\beta_{i-1} \beta_{i} \beta_{l}}\right) .
\end{aligned}
$$

From the first line to the second line, the commutativity of the operators has been used. First, we apply the operators $\mathcal{T}\left[\alpha_{1}, \alpha_{m}\right]$ and $\mathcal{T}\left[\beta_{1}, \beta_{l}\right]$ to $\mathbf{P f}^{\prime} \Psi^{\Lambda}$. Using (43), we obtain

$$
\begin{aligned}
& \mathcal{T}\left[\beta_{1}, \beta_{l}\right] \mathcal{T}\left[\alpha_{1}, \alpha_{m}\right] \mathbf{P f}^{\prime} \Psi^{\Lambda} \\
& =\left(-T_{\beta_{1} \beta_{l}} T_{\beta_{l} \beta_{1}}\right)\left(-T_{\alpha_{1} \alpha_{m}} T_{\alpha_{m} \alpha_{1}}\right) \\
& \quad \times \sum_{\{i, j\}}^{\prime} \mathcal{P}_{\{i, j\}}^{\Lambda}(n, 4,2),
\end{aligned}
$$

where

$$
\begin{aligned}
& \sum_{\{i, j\}}^{\prime} \mathcal{P}_{\{i, j\}}^{\Lambda}(n, 4,2) \\
& \quad=\mathcal{P}_{\{i, j\}}^{\Lambda}(n, 4,2)=\frac{-1}{T_{\beta_{1} \beta_{l}}} \mathbf{P f}[\Psi]_{n-4, \beta_{1}, \beta_{l}: n-4}^{\Lambda} \\
& =\frac{-1}{T_{\alpha_{1} \alpha_{m}}} \operatorname{Pf}[\Psi]_{n-4, \alpha_{1}, \alpha_{m}: n-4}^{\Lambda} .
\end{aligned}
$$

Secondly, we use (49) to get

$$
\begin{aligned}
& \mathcal{I}_{\alpha_{m-2} \alpha_{m-1} \alpha_{m}} \cdots \mathcal{I}_{\alpha_{2} \alpha_{3} \alpha_{m}} \mathcal{I}_{\alpha_{1} \alpha_{2} \alpha_{m}} \\
& \quad \times\left(-T_{\alpha_{1} \alpha_{m}} T_{\alpha_{m} \alpha_{1}}\right) \sum_{\{i, j\}}^{\prime} \mathcal{P}_{\{i, j\}}^{\Lambda}(n, 4,2) \\
& =(-)^{n-4+1} \mathcal{I}_{\alpha_{m-2} \alpha_{m-1} \alpha_{m}} \cdots \mathcal{I}_{\alpha_{2} \alpha_{3} \alpha_{m}} \\
& \quad \times\left(T_{\alpha_{1} \alpha_{2}} T_{\alpha_{2} \alpha_{m}} T_{\alpha_{m} \alpha_{1}}\right) \sum_{\{i, j\}}^{\prime} \mathcal{P}_{\{i, j\}}^{\Lambda}(n, 5,2) \\
& \quad \ldots \\
& =(-)^{\frac{(2 n-5-m)(m-2)}{2}}+1 \mathcal{C}_{\alpha}^{\Lambda} \sum_{\{i, j\}}^{\prime} \mathcal{P}_{\{i, j\}}^{\Lambda}(n, 2+m, 2) .
\end{aligned}
$$

Thirdly, we use (49) again to obtain

$$
\begin{aligned}
& \mathcal{I}_{\beta_{l-2} \alpha_{l-1} \alpha_{l}} \cdots \mathcal{I}_{\beta_{2} \beta_{3} \beta_{l}} \mathcal{I}_{\beta_{1} \beta_{2} \beta_{l}} \\
& \quad \times\left(-T_{\beta_{1} \beta_{l}} T_{\beta_{l} \beta_{1}}\right) \sum_{\{i, j\}}^{\prime} \mathcal{P}_{\{i, j\}}^{\Lambda}(n, 2+m, 2) \\
& =(-)^{\frac{(2 n-2 m-l-1)(l-2)}{2}+1} \mathcal{C}_{\beta}^{\Lambda} \sum_{\{i, j\}}^{\prime} \mathcal{P}_{\{i, j\}}^{\Lambda}(n, l+m, 2) .
\end{aligned}
$$

Combining (70), (72) and (73) together, we get

$$
\begin{aligned}
& \mathcal{T}[\alpha] \cdot \mathcal{T}[\beta] \mathbf{P f}^{\prime} \Psi^{\Lambda}=(-)^{\frac{(2 n-l-m-3)(l+m-4)}{2}+2} \mathcal{C}_{\alpha}^{\Lambda} \mathcal{C}_{\beta}^{\Lambda} \\
& \quad \times \sum_{\{i, j\}}^{\prime} \mathcal{P}_{\{i, j\}}^{\Lambda}(n, l+m, 2) .
\end{aligned}
$$

The most general formula is given by

$$
\begin{aligned}
\mathcal{T} & {\left[\alpha_{1}\right] \cdot \mathcal{T}\left[\alpha_{2}\right] \cdots \mathcal{T}\left[\alpha_{k}\right] \mathbf{P f}^{\prime} \Psi^{\Lambda} } \\
= & (-)^{\frac{\left(2 n-2 k-\sum\left|\alpha_{i}\right|+1\right)\left(\sum\left|\alpha_{i}\right|-2 k\right)}{2}}+k\left(\prod \mathcal{C}_{\alpha_{i}}^{\Lambda}\right) \\
& \times \sum_{\{i, j\}}^{\prime} \mathcal{P}_{\{i, j\}}^{\Lambda}\left(n, \sum\left|\alpha_{i}\right|, k\right),
\end{aligned}
$$

where $\left|\alpha_{i}\right|$ denotes the length of the set $\alpha_{i}$. It can be obtained recursively, by applying an extremely similar technique.

\subsection{Operators $\mathcal{T}[a, b] \cdot \mathcal{L}$ and $\mathcal{T}[a, b] \cdot \widetilde{\mathcal{L}}$}

The operator $\mathcal{L}$ is defined through the longitudinal operators as [6]

$\mathcal{L} \equiv \prod_{i} \mathcal{L}_{i}=\widetilde{\mathcal{L}}+\cdots$, with $\widetilde{\mathcal{L}} \equiv \sum_{\rho \in \text { pair }} \prod_{i_{k}, j_{k} \in \rho} \mathcal{L}_{i_{k} j_{k}}$.

Here the set of pairs $\left\{\left(i_{1}, j_{1}\right),\left(i_{2}, j_{2}\right), \ldots,\left(i_{m}, j_{m}\right)\right\}$ is a partition of $I$ with conditions $i_{1}<i_{2}<\cdots<i_{m}$ and $i_{t}<j_{t}, \forall t$. At the algebraic level, two operators $\mathcal{L}$ and $\widetilde{\mathcal{L}}$ are not equivalent to each other. However, for on-shell integrands, if we apply the combinatory operators $\mathcal{T}[a, b] \cdot \mathcal{L}$ and $\mathcal{T}[a, b] \cdot \widetilde{\mathcal{L}}$ to $\mathbf{P f}^{\prime} \Psi^{\Lambda}$ with even number of external legs, and let subscripts of $\mathcal{L}_{i}$ and $\mathcal{L}_{i j}$ run through all nodes in $\{1,2, \ldots, n\} \backslash\{a, b\}$, the two operators have the same effect [6-8], with

$\left.\mathcal{T}[a, b] \cdot \mathcal{L} \mathbf{P f}^{\prime} \Psi^{\Lambda}=\mathcal{T}[a, b] \cdot \widetilde{\mathcal{L}} \mathbf{P f}^{\prime} \Psi^{\Lambda} \doteq \mathbf{P f}^{\prime} A^{\Lambda}\right)^{2}$, 
up to an overall sign. We now show that Eq. (77) also holds for off-shell integrands.

We first consider the effect of the manipulation $\mathcal{T}[a, b]$. $\widetilde{\mathcal{L}} \mathbf{P} \mathbf{f}^{\prime} \Psi^{\Lambda}$. Acting $\mathcal{T}[a, b]$ on $\mathbf{P f}^{\prime} \Psi^{\Lambda}$ gives (41), which is the reduced Pfaffian of the matrix (39). Using the previous result (55), it is straightforward to see that $\widetilde{\mathcal{L}}$ transmutes the matrix (39) to

$\Psi^{\prime \Lambda}=\left(\begin{array}{c|c|c}A_{n \times n}^{\Lambda} & 0 & 0 \\ \hline 0 & -A_{(n-2) \times(n-2)}^{\Lambda} & 0 \\ \hline 0 & 0 & X_{2 \times 2}^{\Lambda}\end{array}\right)$.

The Pfaffian of the matrix

$$
\left(\begin{array}{c|c}
-A_{(n-2) \times(n-2)}^{\Lambda} & 0 \\
\hline 0 & X_{2 \times 2}^{\Lambda}
\end{array}\right)
$$

is just $-\mathbf{P} \mathbf{f}^{\prime}\left(-A^{\Lambda}\right)=(-)^{\frac{n}{2}} \mathbf{P f}^{\prime} A^{\Lambda}$; thus

$\mathcal{T}[a, b] \cdot \widetilde{\mathcal{L}} \mathbf{P f}^{\prime} \Psi^{\Lambda}=\mathbf{P f}^{\prime} \Psi^{\prime \Lambda}=(-)^{\frac{n}{2}}\left(\mathbf{P f}^{\prime} A^{\Lambda}\right)^{2}$.

Then we consider the effect of acting $\mathcal{L}$ on $\mathcal{T}[a, b] \mathbf{P f}^{\prime} \Psi^{\Lambda}$. Using (59) we know the operator $\mathcal{L}$ transmutes the matrix (39) to

$\Psi^{\prime \prime \Lambda}=\left(\begin{array}{c|c|c}A_{n \times n}^{\Lambda} & A_{n \times(n-2)}^{\Lambda} & 0 \\ \hline A_{(n-2) \times n}^{\Lambda} & 0 & 0 \\ \hline 0 & 0 & X_{2 \times 2}^{\Lambda}\end{array}\right)$,

thus the reduced Pfaffian $\mathbf{P f}^{\prime} \Psi^{\Lambda}$ is turned to

$\mathbf{P f}^{\prime} \Psi^{\prime \prime \Lambda}=\mathbf{P f}^{\prime} \widetilde{A}^{\Lambda} \operatorname{Pf}[X]_{2}^{\Lambda}$,

where

$\widetilde{A}^{\Lambda} \equiv\left(\begin{array}{c|c}A_{n \times n}^{\Lambda} & A_{n \times(n-2)}^{\Lambda} \\ \hline A_{(n-2) \times n}^{\Lambda} & 0\end{array}\right)$.

For simplicity, we choose the $a$ th and $b$ th rows and columns to be removed when evaluating the reduced Pfaffian of $\widetilde{A}^{\Lambda}$. Then we need to compute

$\operatorname{Pf}\left(\begin{array}{c|c}\left(A_{n \times n}^{\Lambda}\right)_{a b}^{a b} & \left(A_{n \times n}^{\Lambda}\right)_{a b}^{a b} \\ \hline\left(A_{n \times n}^{\Lambda}\right)_{a b}^{a b} & 0\end{array}\right)$.

Using the definition of Pfaffian (18), one can find that the non-vanishing contributions for (84) come from rows $i \in$ $\{1, \ldots, n-2\}$ and columns $j \in\{n-1, \ldots, 2 n-4\}$, which give rise to the determinant of the matrix $\left(A^{\Lambda}\right)_{a b}^{a b}$. Thus the reduced Pfaffian of $\widetilde{A}^{\Lambda}$ can be obtained:

$$
\begin{aligned}
\mathbf{P f}^{\prime} \widetilde{A}^{\Lambda} & =(-)^{\frac{(n-2)(n-3)}{2}+1} T_{a b} \operatorname{det}\left(A^{\Lambda}\right)_{a b}^{a b} \\
& =(-)^{\frac{n}{2}} \frac{1}{T_{a b}}\left(\mathbf{P f}^{\prime} A^{\Lambda}\right)^{2},
\end{aligned}
$$

where we have used $(-)^{\frac{(n-2)(n-3)}{2}}=(-)^{\frac{n-2}{2}}$, due to the fact that $n$ is even. Putting it back to (82), we obtain

$\mathcal{T}[a, b] \cdot \mathcal{L} \mathbf{P f}^{\prime} \Psi^{\Lambda}=\mathbf{P f}^{\prime} \Psi^{\prime \prime \Lambda}=(-)^{\frac{n}{2}}\left(\mathbf{P f}^{\prime} A^{\Lambda}\right)^{2}$.
The above calculations show that

$$
\begin{aligned}
\mathcal{T}[a, b] \cdot \mathcal{L} \mathbf{P f}^{\prime} \Psi^{\Lambda} & =\mathcal{T}[a, b] \cdot \widetilde{\mathcal{L}} \mathbf{P f}^{\prime} \Psi^{\Lambda} \\
& =(-)^{\frac{n}{2}}\left(\mathbf{P f}^{\prime} A^{\Lambda}\right)^{2}
\end{aligned}
$$

It is worth to notice that this result is independent of the choice of $a$ and $b$.

Although Eq. (77) for on-shell amplitudes also holds for off-shell ones, we must emphasize that the definitions of the operators $\mathcal{L}$ and $\widetilde{\mathcal{L}}$ are different for the on-shell and off-shell cases, since for the off-shell case, corrections $\Delta_{i j}$ are introduced when defining longitudinal operators $\mathcal{L}_{i}$ and $\mathcal{L}_{i j}$. We have shown that, with the redefined $\mathcal{L}_{i}$ and $\mathcal{L}_{i j}$, one can transmute $\mathbf{P} \mathbf{f}^{\prime} \Psi^{\Lambda}$ to $\left(\mathbf{P f ^ { \prime }} A^{\Lambda}\right)^{2}$, which serves as a building block for the BI, DBI, exDBI, NLSM and SG integrands, and therefore lead to a meaningful interpretation. If we insist on the original definition, the resulting final object cannot be interpreted physically.

\subsection{Operators $\mathcal{T}_{X_{2 m}}$ and $\mathcal{T}_{\mathcal{X}_{2 m}}$}

In this subsection, we consider the combinatory operators $\mathcal{T}_{X_{2 m}}$ and $\mathcal{T}_{\mathcal{X}_{2 m}}$, which generate $\operatorname{Pf}[X]_{2 m}^{\Lambda}$ and $\operatorname{Pf}[\mathcal{X}]_{2 m}^{\Lambda}$ from $\mathbf{P f}^{\prime} \Psi^{\Lambda}$, respectively.

For a given length-2m set $I$, the operator $\mathcal{T}_{X_{2 m}}$ is defined as

$\mathcal{T}_{X_{2 m}} \equiv \sum_{\rho \in \text { pair }} \prod_{i_{k}, j_{k} \in \rho} \mathcal{T}\left[i_{k}, j_{k}\right]$.

The notation $\sum_{\rho \in \text { pair }} \prod_{i_{k}, j_{k} \in \rho}$ is explained after (76). Using the result in (43) and the definition of Pfaffian (18), one can find that the operator $\mathcal{T}_{X_{2 m}}$ transmutes $\Psi^{\Lambda}$ to a new matrix

$\widetilde{\Psi}^{* \Lambda}=\left(\begin{array}{c|c|c}A_{n \times n}^{\Lambda} & C_{n \times(n-2 m)}^{\Lambda} & 0 \\ \hline-\left(C^{\Lambda}\right)_{(n-2 m) \times n}^{\mathrm{T}} & B_{(n-2 m) \times(n 2-m)}^{\Lambda} & 0 \\ \hline 0 & 0 & X_{2 m \times 2 m}^{\Lambda}\end{array}\right)$,

so that

$\mathcal{T}_{X_{2 m}} \mathbf{P f}^{\prime} \Psi^{\Lambda}=\mathbf{P f}^{\prime} \widetilde{\Psi}^{* \Lambda}=\mathbf{P f}^{\prime}[\Psi]_{n-2 m, 2 m: n-2 m}^{\Lambda} \mathbf{P f}[X]_{2 m}^{\Lambda}$,

therefore providing the building block $\mathbf{P f}[X]_{2 m}^{\Lambda}$.

The operator $\mathcal{T}_{\mathcal{X}_{2 m}}$ is defined in a similar form:

$\mathcal{T}_{\mathcal{X}_{2 m}} \equiv \sum_{\rho \in \text { pair }} \prod_{i_{k}, j_{k} \in \rho} \delta^{I_{i_{k}}, I_{j_{k}}} \mathcal{T}\left[i_{k}, j_{k}\right]$

where $\delta^{I_{i_{k}}, I_{j_{k}}}$ forbids the interaction between particles with different flavors. These $\delta^{I_{i_{k}}, I_{j_{k}}}$ turn the matrix $[X]_{2 m}^{\Lambda}$ to $[\mathcal{X}]_{2 m}^{\Lambda}$. Thus, we have

$\mathcal{T}_{\mathcal{X}_{2 m}} \mathbf{P f}^{\prime} \Psi^{\Lambda}=\mathbf{P f}^{\prime}[\Psi]_{n-2 m, 2 m: n-2 m}^{\Lambda} \mathbf{P f}[\mathcal{X}]_{2 m}^{\Lambda}$,

which gives the building block $\operatorname{Pf}[\mathcal{X}]_{2 m}^{\Lambda}$. 


\section{Relations among amplitudes}

With the preparations in the previous sections, we are ready to exhibit relations among different off-shell amplitudes. We will re-establish the unified set in [6] for off-shell amplitudes. Then we will claim that the three important relations among color-ordered on-shell amplitudes, including the color-ordered reversed relation, the photon decoupling relation, and the KK relation, can be generalized to the off-shell case.

\subsection{Transmuting amplitudes by differential operators}

In this subsection, we will show that the combinatory differential operators discussed in Sect. 4 transmute the GR amplitude to amplitudes of a variety of other theories. It is quite natural to take the GR amplitude whose external states carry the highest spins as the starting point, since all operators decrease the spins of the external legs. The GR integrand in the single-cover formula is shown in the first line of Table 1. To obtain the double-cover expression, we can get $\mathcal{I}_{L}^{\Lambda}$ and $\mathcal{I}_{R}^{\Lambda}$ from $\mathcal{I}_{L}$ and $\mathcal{I}_{R}$, via the replacement $\frac{1}{z_{i j}} \rightarrow T_{i j}$, then multiplying both of them by the factor $\left(\prod_{i=1}^{n} \frac{(y \sigma)_{i}}{y_{i}}\right)$. Thus, we have

$\mathcal{A}_{\mathrm{GR}}=\int d \mu_{n}^{\Lambda}\left(\prod_{i=1}^{n} \frac{(y \sigma)_{i}}{y_{i}}\right)^{2} \mathbf{P f}^{\prime} \Psi^{\Lambda} \mathbf{P f}^{\prime} \widetilde{\Psi}^{\Lambda}$.

We will consider the effect of applying differential operators to the above GR CHY integral. As introduced in Sect. 1, the key point is that the differential operators are commutable with the integral over auxiliary coordinates; thus, transmuting an amplitude is equivalent to transmuting the corresponding $\mathrm{CHY}$ integrand. Furthermore, since differential operators do not affect the factor $\frac{(y \sigma)_{i}}{y_{i}}$, transmuting $\mathcal{I}_{L}^{\tau}$ or $\mathcal{I}_{R}^{\tau}$ is equivalent to transmuting $\mathcal{I}_{L}^{\Lambda}$ or $\mathcal{I}_{R}^{\Lambda}$. In summary, we have

$$
\begin{aligned}
\mathcal{O} \mathcal{A} & =\mathcal{O} \int d \mu_{n}^{\Lambda}\left(\prod_{i=1}^{n} \frac{(y \sigma)_{i}}{y_{i}}\right)^{2} \mathcal{I}_{L}^{\Lambda} \mathcal{I}_{R}^{\Lambda} \\
& =\int d \mu_{n}^{\Lambda}\left(\prod_{i=1}^{n} \frac{(y \sigma)_{i}}{y_{i}}\right)^{2}\left(\mathcal{O} \mathcal{I}_{L}^{\Lambda} \mathcal{I}_{R}^{\Lambda}\right) .
\end{aligned}
$$

In the GR integrand, two parts $\mathcal{I}_{L}^{\Lambda}$ and $\mathcal{I}_{R}^{\Lambda}$ depend on two independent sets of polarization vectors $\left\{\epsilon_{i}\right\}$ and $\left\{\tilde{\epsilon}_{i}\right\}$, respectively. It means that we can define two independent sets of differential operators, through two sets of polarization vectors. The operators defined via $\left\{\epsilon_{i}\right\}$ only act on $\mathcal{I}_{L}^{\Lambda}$, while the operators defined via $\left\{\widetilde{\epsilon}_{i}\right\}$ only act on $\mathcal{I}_{R}^{\Lambda}$. This property protects the manifest double copy structure of $\mathrm{CHY}$ integrands. Without loss of generality, we can restrict our consideration to the effect of operators on the $\mathcal{I}_{L}^{\Lambda}$ part, by defining them via $\left\{\epsilon_{i}\right\}$.
Performing operators on the $\mathcal{I}_{L}^{\Lambda}$ part and using (43), (65), (75), (87), (90), and (92), after comparing with the middle column of Table 1, we get the following relations:

$$
\begin{aligned}
\mathcal{A}_{\mathrm{EYM}} & =\mathcal{T}\left[\operatorname{Tr}_{1}\right] \cdots \mathcal{T}\left[\operatorname{Tr}_{m}\right] \mathcal{A}_{\mathrm{GR}}, \\
\mathcal{A}_{\mathrm{YM}} & =\mathcal{T}\left[i_{1} \cdots i_{n}\right] \mathcal{A}_{\mathrm{GR}} \\
\mathcal{A}_{\mathrm{EM}} & =\mathcal{T}_{X_{2 m}} \mathcal{A}_{\mathrm{GR}} \\
\mathcal{A}_{\mathrm{EMf}} & =\mathcal{T}_{\mathcal{X}_{2 m}} \mathcal{A}_{\mathrm{GR}} \\
\mathcal{A}_{\mathrm{BI}} & =\mathcal{T}[a, b] \cdot \mathcal{L} \mathcal{A}_{\mathrm{GR}}
\end{aligned}
$$

up to an overall sign.

Similarly, applying operators to the pure YM integrand, we obtain the relations

$$
\begin{aligned}
\mathcal{A}_{\mathrm{YMS}} & =\mathcal{T}\left[\operatorname{Tr}_{1}\right] \cdots \mathcal{T}\left[\operatorname{Tr}_{m}\right] \mathcal{A}_{\mathrm{YM}} \\
\mathcal{A}_{\mathrm{SYMS}} & =\mathcal{T}_{\mathcal{X}_{2 m}} \mathcal{A}_{\mathrm{YM}} \\
\mathcal{A}_{\mathrm{BAS}} & =\mathcal{T}\left[i_{1} \cdots i_{n}\right] \mathcal{A}_{\mathrm{YM}} \\
\mathcal{A}_{\mathrm{NLSM}} & =\mathcal{T}[a, b] \cdot \mathcal{L} \mathcal{A}_{\mathrm{YM}} \\
\mathcal{A}_{\phi^{4}} & =\mathcal{T}_{X_{n}} \mathcal{A}_{\mathrm{YM}}
\end{aligned}
$$

up to an overall sign. Notice that the amplitude of $\phi^{4}$ theory is generated via the particular entity $\mathcal{T}_{X_{2 m}}$ with $2 m=n$.

Applying operators to the BI integrand, we get the relations

$$
\begin{aligned}
\mathcal{A}_{\mathrm{exDBI}} & =\mathcal{T}\left[\operatorname{Tr}_{1}\right] \cdots \mathcal{T}\left[\operatorname{Tr}_{m}\right] \mathcal{A}_{\mathrm{BI}}, \\
\mathcal{A}_{\mathrm{DBI}} & =\mathcal{T}_{\mathcal{X}_{2 m}} \mathcal{A}_{\mathrm{BI}}, \\
\mathcal{A}_{\mathrm{NLSM}} & =\mathcal{T}\left[i_{1} \cdots i_{n}\right] \mathcal{A}_{\mathrm{BI}}, \\
\mathcal{A}_{\mathrm{SG}} & =\mathcal{T}[a, b] \cdot \mathcal{L} \mathcal{A}_{\mathrm{BI}},
\end{aligned}
$$

up to an overall sign.

The relations presented in (95), (96) and (97) can be organized as

$\mathcal{A}_{\text {other }}=\mathcal{O}^{\epsilon} \cdot \mathcal{O}^{\tilde{\epsilon}} \mathcal{A}_{\mathrm{G}}^{\epsilon, \tilde{\epsilon}}$

where $\mathcal{O}^{\epsilon}$ and $\mathcal{O}^{\tilde{\epsilon}}$ denote operators which are defined through $\left\{\epsilon_{i}\right\}$ and $\left\{\tilde{\epsilon}_{i}\right\}$, respectively. The corresponding operators $\mathcal{O}^{\epsilon}$ and $\mathcal{O}^{\tilde{\epsilon}}$ for different theories are listed in Table 2.

In this table, $\mathbb{I}$ denotes the identical operator. As pointed out before, because of the manifest double copy structure of the $\mathrm{CHY}$ integrands, $\mathcal{O}^{\epsilon}$ and $\mathcal{O}^{\tilde{\epsilon}}$ act on two pieces independently at the integrand-level.

From the relations mentioned in Table 2, various other relations can be extracted. For example, substituting

$\mathcal{A}_{\mathrm{EYM}}^{\epsilon, \tilde{\epsilon}}=\mathcal{T}^{\epsilon}\left[\operatorname{Tr}_{1}\right] \cdots \mathcal{T}^{\epsilon}\left[\operatorname{Tr}_{m}\right] \mathcal{A}_{\mathrm{GR}}^{\epsilon, \tilde{\epsilon}}$

into

$$
\begin{aligned}
\mathcal{A}_{\mathrm{YMS}}^{\epsilon}= & \left(\mathcal{T}^{\epsilon}\left[\operatorname{Tr}_{1}\right] \cdots \mathcal{T}^{\epsilon}\left[\operatorname{Tr}_{m}\right]\right) \\
& \cdot \mathcal{T}^{\widetilde{\epsilon}}\left[i_{1}, \ldots, i_{n}\right] \mathcal{A}_{\mathrm{GR}}^{\epsilon, \tilde{\epsilon}},
\end{aligned}
$$

we get

$\mathcal{A}_{\mathrm{YMS}}^{\epsilon}=\mathcal{T}^{\tilde{\epsilon}}\left[i_{1}, \ldots, i_{n}\right] \mathcal{A}_{\mathrm{EYM}}^{\epsilon, \tilde{\epsilon}}$ 
Table 2 Unifying relations

\begin{tabular}{lll}
\hline Amplitude & $\mathcal{O}^{\epsilon}$ & $\mathcal{O}^{\tilde{\epsilon}}$ \\
\hline $\mathcal{A}_{\mathrm{EYM}}^{\epsilon, \tilde{\epsilon}}$ & $\mathcal{T}^{\epsilon}\left[\operatorname{Tr}_{1}\right] \ldots \mathcal{T}^{\epsilon}\left[\operatorname{Tr}_{m}\right]$ & $\mathbb{I}$ \\
$\mathcal{A}_{\mathrm{YM}}^{\tilde{\epsilon}}$ & $\mathcal{T}^{\epsilon}\left[i_{1}, \ldots, i_{n}\right]$ & $\mathbb{I}$ \\
$\mathcal{A}_{\mathrm{EM}}^{\epsilon, \tilde{\epsilon}}$ & $\mathcal{T}_{X_{2 m}^{\epsilon}}^{\epsilon}$ & $\mathbb{I}$ \\
$\mathcal{A}_{\mathrm{EMf}}^{\epsilon, \tilde{\epsilon}}$ & $\mathcal{T}_{\mathcal{X}_{2 m}}^{\epsilon}$ & $\mathbb{I}$ \\
$\mathcal{A}_{\mathrm{BI}}^{\tilde{\epsilon}}$ & $\mathcal{L}^{\epsilon} \cdot \mathcal{T}^{\epsilon}[a b]$ & $\mathbb{I}$ \\
$\mathcal{A}_{\mathrm{YMS}}^{\tilde{\epsilon}}$ & $\mathcal{T}^{\epsilon}\left[i_{1}, \ldots, i_{n}\right]$ & $\mathcal{T}^{\tilde{\epsilon}}\left[\operatorname{Tr}_{1}\right] \cdots \mathcal{T}^{\tilde{\epsilon}}\left[\operatorname{Tr}_{m}\right]$ \\
$\mathcal{A}_{\mathrm{SYMS}}^{\widetilde{\epsilon}}$ & $\mathcal{T}^{\epsilon}\left[i_{1}, \ldots, i_{n}\right]$ & $\mathcal{T}_{\mathcal{X}_{2 m}}^{\tilde{\epsilon}}$ \\
$\mathcal{A}_{\mathrm{BAS}}$ & $\mathcal{T}^{\epsilon}\left[i_{1}, \ldots, i_{n}\right]$ & $\mathcal{T}^{\tilde{\epsilon}}\left[i_{1}^{\prime}, \ldots, i_{n}^{\prime}\right]$ \\
$\mathcal{A}_{\mathrm{NLSM}}$ & $\mathcal{T}^{\epsilon}\left[i_{1}, \ldots, i_{n}\right]$ & $\mathcal{L}^{\tilde{\epsilon}} \cdot \mathcal{T}_{a^{\prime} b^{\prime}}^{\tilde{\epsilon}}$ \\
$\mathcal{A}_{\phi^{4}}$ & $\mathcal{T}^{\epsilon}\left[i_{1}, \ldots, i_{n}\right]$ & $\mathcal{T}_{X_{n}}^{\tilde{\epsilon}}$ \\
$\mathcal{A}_{\mathrm{exDBI}}^{\tilde{\epsilon}}$ & $\mathcal{L}^{\epsilon} \cdot \mathcal{T}^{\epsilon}[a b]$ & $\mathcal{T}^{\tilde{\epsilon}}\left[\operatorname{Tr}_{1}\right] \cdots \mathcal{T}^{\tilde{\epsilon}}\left[\operatorname{Tr}_{m}\right]$ \\
$\mathcal{A}_{\mathrm{DBI}}^{\tilde{\epsilon}}$ & $\mathcal{L}^{\epsilon} \cdot \mathcal{T}^{\epsilon}[a b]$ & $\mathcal{T}_{\mathcal{X}_{2 m}}^{\tilde{\epsilon}}$ \\
$\mathcal{A}_{\mathrm{SG}}$ & $\mathcal{L}^{\epsilon} \cdot \mathcal{T}^{\epsilon}[a b]$ & $\mathcal{L}^{\tilde{\epsilon}} \cdot \mathcal{T}^{\tilde{\epsilon}}\left[a^{\prime} b^{\prime}\right]$ \\
\hline & & \\
\hline & &
\end{tabular}

Differential operators connect not only amplitudes of different theories, but also amplitudes of the same theory. For example, using Eq. (49) one can get

$$
\begin{aligned}
& \mathcal{A}_{\mathrm{EYM}}^{\epsilon, \tilde{\epsilon}}\left(\left\{i_{3}^{h}, \ldots, i_{m}^{h}\right\} \| j_{1}^{g}, i_{1}^{g}, i_{2}^{g}, j_{2}^{g}, \ldots, j_{n}^{g}\right) \\
& =\mathcal{T}_{i_{1} i_{2} j_{2}}^{\epsilon} \mathcal{T}_{j_{1} i_{1} j_{2}}^{\epsilon} \mathcal{A}_{\mathrm{EYM}}^{\epsilon, \widetilde{\epsilon}}\left(\left\{i_{1}^{h}, \ldots, i_{m}^{h}\right\} \| j_{1}^{g}, \ldots, j_{n}^{g}\right) .
\end{aligned}
$$

The notation $\left(\left\{i_{1}^{h}, \ldots, i_{m}^{h}\right\} \| j_{1}^{g}, \ldots, j_{n}^{g}\right)$ means there is no color-ordering among elements in the set $\left\{i_{1}^{h}, \ldots, i_{m}^{h}\right\}$, while the elements $\left(j_{1}^{g}, \ldots, j_{n}^{g}\right)$ are color-ordered. The insertion operators turn gravitons $i_{1}^{h}$ and $i_{2}^{h}$ to gluons, and insert them between gluons $j_{1}^{g}$ and $j_{2}^{g}$ in the color-ordering $\left(j_{1}^{g}, \ldots, j_{n}^{g}\right)$. Consequently, the insertion operators $\mathcal{T}_{i_{1} i_{2} j_{2}}^{\epsilon}, \mathcal{T}_{j_{1} i_{1} j_{2}}^{\epsilon}$ transmute the EYM amplitude $\mathcal{A}_{\mathrm{EYM}}^{\epsilon, \tilde{\epsilon}}\left(\left\{i_{1}^{h}, \ldots, i_{m}^{h}\right\} \| j_{1}^{g}, \ldots, j_{n}^{g}\right)$ to the EYM amplitude $\mathcal{A}_{\mathrm{EYM}}^{\epsilon, \tilde{\epsilon}}\left(\left\{i_{3}^{h}, \ldots, i_{m}^{h}\right\} \| j_{1}^{g}, i_{1}^{g}, i_{2}^{g}, j_{2}^{g}\right.$, $\left.\ldots, j_{n}^{g}\right)$.

\subsection{Three generalized relations for color-ordered amplitudes}

In this subsection, we demonstrate that the three generalized relations for on-shell color-ordered amplitudes also hold for off-shell amplitudes. From Table 2, one can see that each color-ordered amplitude can be generated by applying the general trace operator $\mathcal{T}[\alpha]$, formally expressed as

$\mathcal{A}\left(i_{1}, \ldots, i_{n}\right)=\mathcal{T}\left[i_{1}, \ldots, i_{n}\right] \mathcal{A}^{\prime}$.

Thus, we will derive three relations by using the algebraic property of the general trace operator.

The first relation is the generalized color-ordered reversed relation for off-shell color-ordered amplitudes:

$\mathcal{A}(1,2, \ldots, n)=(-)^{n} \mathcal{A}(n, n-1, \ldots, 1)$.
The color-ordering $(1,2, \ldots, n ; \ldots)$ can be created by the general trace operator

$\mathcal{T}[1,2, \ldots, n]=\mathcal{T}[1, n] \cdot \mathcal{I}_{12 n} \cdot \mathcal{I}_{23 n} \cdots \mathcal{I}_{(n-2)(n-1) n}$.

Using $\mathcal{T}[1, n]=\mathcal{T}[n, 1]$ and $\mathcal{I}_{i k j}=-\mathcal{I}_{j k i}$, we have

$$
\begin{aligned}
\mathcal{T}[1,2, \ldots, n] & =(-)^{n-2} \mathcal{T}[n, 1] \cdot \mathcal{I}_{n 21} \cdot \mathcal{I}_{n 32} \cdots \mathcal{I}_{n(n-1)(n-2)} \\
& =(-)^{n} \mathcal{T}[n, n-1, \ldots, 1]
\end{aligned}
$$

which immediately gives rise to (104).

The second relation is the generalized photon decoupling relation. Using the definition of the insertion operators, one can decompose $\mathcal{I}_{1 h n}$ as

$\mathcal{I}_{1 h n}=\mathcal{I}_{1 h 2}+\mathcal{I}_{2 h 3}+\cdots+\mathcal{I}_{(n-1) h n}$,

therefore

$\mathcal{I}_{1 h 2}+\mathcal{I}_{2 h 3}+\cdots+\mathcal{I}_{(n-1) h n}+\mathcal{I}_{1 h n}=0$.

This algebraic relation indicates that

$\sum_{\uplus} \mathcal{A}(1, h \amalg\{2, \ldots, n\})=0$.

The shuffling of two ordered sets $\alpha \sqcup \beta$ is a permutation of the set $\alpha \cup \beta$ while preserving the ordering of $\alpha$ and $\beta$. For instance,

$$
\begin{aligned}
& \sum_{\uplus}\{1,2\} \amalg\{3,4\}=\{1,2,3,4\}+\{1,3,2,4\}+\{1,3,4,2\} \\
& \quad+\{3,1,2,4\}+\{3,1,4,2\}+\{3,4,1,2\} .
\end{aligned}
$$

The third relation is the generalized Kleiss-Kuijf (KK) relation

$\mathcal{A}(1, \alpha, n, \beta)=\sum_{\amalg}(-)^{|\beta|} \mathcal{A}\left(1, \alpha \amalg \beta^{T}, n\right)$,

where $\alpha$ and $\beta$ are two ordered sets, and $\beta^{T}$ is obtained from $\beta$ by reversing the ordering of elements. To derive it, let us reformulate the trace operator $\mathcal{T}\left[a_{1}, \ldots, a_{n}\right]$ as

$$
\begin{aligned}
\mathcal{T}\left[a_{1}, \ldots, a_{n}\right]= & \mathcal{T}\left[a_{1}, a_{n}\right] \cdot \prod_{i=2}^{n-1} \mathcal{I}_{a_{i-1} a_{i} a_{n}} \\
= & \mathcal{T}\left[a_{1}, a_{n}\right] \cdot\left(\prod_{i=1}^{k} \mathcal{I}_{a_{i-1} a_{i} a_{n}}\right) \\
& \cdot\left((-)^{n-k-1} \prod_{j=k+1}^{n-1} \mathcal{I}_{a_{n} a_{j} a_{j-1}}\right) .
\end{aligned}
$$

The operator $\mathcal{T}\left[a_{1}, a_{n}\right] \cdot\left(\prod_{i=1}^{k} \mathcal{I}_{a_{i-1} a_{i} a_{n}}\right)$ generates the color-ordering $\left(a_{1}, a_{2}, \ldots, a_{k}, a_{n}\right)$, which is equivalent to $\left(a_{n}, a_{1}, \ldots, a_{k}\right)$, due to the cyclic symmetry. The operator 
$\left((-)^{n-k-1} \prod_{j=k+1}^{n-1} \mathcal{I}_{a_{n} a_{j} a_{j-1}}\right)$ can be interpreted as inserting $\left\{a_{n-1}, a_{n-2}, \ldots, a_{k+1}\right\}$ between $a_{n}$ and $a_{k}$. More explicitly, using the definition of the insertion operator we know that

$\mathcal{I}_{i k j}=\mathcal{I}_{i k l}+\mathcal{I}_{l k j}$

Repeating this decomposition, we find that the operator $\mathcal{I}_{a_{n} a_{k+1} a_{k}}$ gives rise to

$\sum_{\uplus} \mathcal{A}\left(a_{n},\left\{a_{1}, \ldots, a_{k-1}\right\} \amalg a_{k+1}, a_{k}\right)$.

Applying a similar procedure recursively, it is easy to see that the operator $\left((-)^{n-k-1} \prod_{j=k+1}^{n-1} \mathcal{I}_{a_{n} a_{j} a_{j-1}}\right)$ leads to

$\sum_{\sqcup} \mathcal{A}\left(a_{n},\left\{a_{1}, \ldots, a_{k-1}\right\} \sqcup\left\{a_{n-1}, \ldots, a_{k+1}\right\}, a_{k}\right)$.

Using the above conclusion, and setting $a_{n}=1, a_{k}=n$, $\left\{a_{1}, \ldots, a_{k-1}\right\}=\alpha,\left\{a_{k+1}, \ldots, a_{n-1}\right\}=\beta$, the KK relation (111) can be observed to hold directly.

\section{Discussion}

By modifying the definition of the longitudinal operators, we have generalized various relations for on-shell amplitudes to off-shell ones. The CHY formalism serves as a powerful tool for this work. As explained in Sect. 1, our result also provides a verification for the double-cover construction.

At the end of the paper, we want to point out three issues.

First, the expansions of on-shell amplitudes, which can be derived via relations in Table 2, cannot be generalized to the off-shell case, since in the derivation proposed in [9-11], the gauge invariance is a necessary tool. As mentioned in Sect. 2, for the off-shell case, the gauge invariance is lost. For some special cases, for example the GR amplitude including two massive external legs with the same mass (a special case of the off-shell external massless states), one can avoid the appearing of corrections $\Delta_{i j}$ and $\eta_{i j}$ by choosing the removed rows and columns in the reduced matrix to be two massive ones, and obtain a reduced matrix that is totally the same as that for the massless on-shell amplitude. Then one can claim that the expansion of the on-shell massless GR amplitude to BAS amplitudes also holds for this massive (or off-shell) GR amplitude, since from the $\mathrm{CHY}$ point of view, this expansion is just expanding $\mathbf{P f}^{\prime} \Psi^{\Lambda}$ to Parke-Taylor factors $\mathcal{C}_{n}^{\Lambda}(\sigma)$. But this manipulation cannot be generalized to general off-shell amplitudes.

Secondly, the differential operators discussed in this paper will not affect any $k_{i}^{2}$. This fact has a physical meaning when treating amplitudes with massive external states as off-shell CHY integrals. Thus, for the massive amplitudes of different theories related by differential operators, the mass for a particular external state is unique, due to the invariance of $k_{i}^{2}$. For example, suppose the leg 1 is a vector particle in the amplitude $\mathcal{A}_{1}$, and is a scalar particle in the amplitude $\mathcal{A}_{2}$. If $\mathcal{A}_{1}$ and $\mathcal{A}_{2}$ are related by a differential operator, then the vector particle and the scalar particle denoted by 1 have the same mass.

Thirdly, in the literature [6], the gauge invariance is one of the conditions for constructing differential operators. In the off-shell case, the gauge invariance is lost. However, differential operators transmute off-shell amplitudes in the same manner as transmuting on-shell ones. It implies that maybe it is not suitable to regard the gauge invariance as the fundamental principle for constructing differential operators. The underlying principle which determines differential operators discussed in this paper is an interesting question.

Acknowledgements This work is supported by Chinese NSF funding under Contracts No. 11805163 and NSF of Jiangsu Province under Grant No. BK20180897.

Data Availability Statement This manuscript has no associated data or the data will not be deposited. [Authors' comment: This research is theoretical and formal.]

Open Access This article is licensed under a Creative Commons Attribution 4.0 International License, which permits use, sharing, adaptation, distribution and reproduction in any medium or format, as long as you give appropriate credit to the original author(s) and the source, provide a link to the Creative Commons licence, and indicate if changes were made. The images or other third party material in this article are included in the article's Creative Commons licence, unless indicated otherwise in a credit line to the material. If material is not included in the article's Creative Commons licence and your intended use is not permitted by statutory regulation or exceeds the permitted use, you will need to obtain permission directly from the copyright holder. To view a copy of this licence, visit http://creativecomm ons.org/licenses/by/4.0/.

Funded by $\mathrm{SCOAP}^{3}$.

\section{References}

1. F. Cachazo, S. He, E.Y. Yuan, Scattering equations and KawaiLewellen-Tye orthogonality. Phys. Rev. D 90(6), 065001 (2014). https://doi.org/10.1103/PhysRevD.90.065001. arXiv:1306.6575 [hep-th]

2. F. Cachazo, S. He, E.Y. Yuan, Scattering of massless particles in arbitrary dimensions. Phys. Rev. Lett. 113(17), 171601 (2014). https://doi.org/10.1103/PhysRevLett.113.171601. arXiv:1307.2199 [hep-th]

3. F. Cachazo, S. He, EY. Yuan, Scattering of massless particles: scalars, gluons and gravitons. JHEP 1407, 033 (2014). https://doi. org/10.1007/JHEP07(2014)033. arXiv:1309.0885 [hep-th]

4. F. Cachazo, S. He, E.Y. Yuan, Einstein-Yang-Mills scattering amplitudes from scattering equations. JHEP 1501, 121 (2015). https://doi.org/10.1007/JHEP01(2015)121. arXiv:1409.8256 [hep-th]

5. F. Cachazo, S. He, E.Y. Yuan, Scattering equations and matrices: from Einstein To Yang-Mills, DBI and NLSM. JHEP 1507, 149 (2015). https://doi.org/10.1007/JHEP07(2015)149. arXiv: 1412.3479 [hep-th] 
6. C. Cheung, C.H. Shen, C. Wen, Unifying relations for scattering amplitudes. JHEP 1802, 095 (2018). https://doi.org/10.1007/ JHEP02(2018)095. arXiv:1705.03025 [hep-th]

7. K. Zhou, B. Feng, Note on differential operators, CHY integrands, and unifying relations for amplitudes. JHEP 1809, 160 (2018). arXiv:1808.06835 [hep-th]

8. M. Bollmann, L. Ferro, Transmuting CHY formulae. JHEP 1901, 180 (2019). arXiv: 1808.07451 [hep-th]

9. B. Feng, X. Li, K. Zhou, Expansion of Einstein-YangMills theory by differential operators. Phys. Rev. D 100(12), 125012 (2019). https://doi.org/10.1103/PhysRevD.100.125012. arXiv:1904.05997 [hep-th]

10. S.Q. Hu, K. Zhou, Expansion of tree amplitudes for EM and other theories. arXiv:1907.07857 [hep-th]

11. K. Zhou, Unified web for expansions of amplitudes. JHEP 1910, 195 (2019). https://doi.org/10.1007/JHEP10(2019)195. arXiv:1908.10272 [hep-th]

12. S. Stieberger, T.R. Taylor, New relations for Einstein-Yang-Mills amplitudes. Nucl. Phys. B 913, 151 (2016). arXiv:1606.09616 [hep-th]

13. O. Schlotterer, Amplitude relations in heterotic string theory and Einstein-Yang-Mills. JHEP 1611, 074 (2016). arXiv:1608.00130 [hep-th]

14. M. Chiodaroli, M. Gunaydin, H. Johansson, R. Roiban, Explicit formulae for Yang-Mills-Einstein amplitudes from the double copy. JHEP 1707, 002 (2017). https://doi.org/10.1007/ JHEP07(2017)002. arXiv:1703.00421 [hep-th]

15. V. Del Duca, L.J. Dixon, F. Maltoni, New color decompositions for gauge amplitudes at tree and loop level. Nucl. Phys. B 571, 51 (2000). https://doi.org/10.1016/S0550-3213(99)00809-3. arXiv:hep-ph/9910563

16. D. Nandan, J. Plefka, O. Schlotterer, C. Wen, Einstein-YangMills from pure Yang-Mills amplitudes. JHEP 1610, 070 (2016). arXiv:1607.05701 [hep-th]

17. L. de la Cruz, A. Kniss, S. Weinzierl, Relations for Einstein-YangMills amplitudes from the CHY representation. Phys. Lett. B 767, 86 (2017). arXiv:1607.06036 [hep-th]

18. C.H. Fu, Y.J. Du, R. Huang, B. Feng, Expansion of Einstein-YangMills amplitude. JHEP 1709, 021 (2017). arXiv:1702.08158 [hepth]
19. F. Teng, B. Feng, Expanding Einstein-Yang-Mills by Yang-Mills in CHY frame. JHEP 1705, 075 (2017). arXiv: 1703.01269 [hep-th]

20. Y.J. Du, F. Teng, BCJ numerators from reduced Pfaffian. JHEP 1704, 033 (2017). arXiv:1703.05717 [hep-th]

21. Y.J. Du, B. Feng, F. Teng, Expansion of all multitrace tree level EYM amplitudes. JHEP 1712, 038 (2017). arXiv:1708.04514 [hepth]

22. H. Gomez, $\Lambda$ scattering equations. JHEP 1606, 101 (2016). https:// doi.org/10.1007/JHEP06(2016)101. arXiv:1604.05373 [hep-th]

23. C. Cardona, H. Gomez, Elliptic scattering equations. JHEP 1606, 094 (2016). https://doi.org/10.1007/JHEP06(2016)094. arXiv:1605.01446 [hep-th]

24. N.E.J. Bjerrum-Bohr, P.H. Damgaard, H. Gomez, New factorization relations for Yang Mills amplitudes. Phys. Rev. D 99(2), 025014 (2019). https://doi.org/10.1103/PhysRevD.99. 025014. arXiv:1810.05023 [hep-th]

25. H. Gomez, Scattering equations and a new factorization for amplitudes. Part I. Gauge theories. JHEP 1905, 128 (2019). https://doi. org/10.1007/JHEP05(2019)128. arXiv:1810.05407 [hep-th]

26. N.E.J. Bjerrum-Bohr, H. Gomez, A. Helset, New factorization relations for nonlinear sigma model amplitudes. Phys. Rev. D 99(4), 045009 (2019). https://doi.org/10.1103/PhysRevD.99. 045009. arXiv:1811.06024 [hep-th]

27. H. Gomez, A. Helset, Scattering equations and a new factorization for amplitudes. Part II. Effective field theories. JHEP 1905, 129 (2019). https://doi.org/10.1007/JHEP05(2019)129. arXiv: 1902.02633 [hep-th]

28. S.G. Naculich, CHY representations for gauge theory and gravity amplitudes with up to three massive particles. JHEP 05, 050 (2015). https://doi.org/10.1007/JHEP05(2015)050. arXiv: 1501.03500 [hep-th]

29. C. Lam, Off-shell Yang-Mills amplitude in the Cachazo-He-Yuan formalism. Phys. Rev. D 100(4,) 045009 (2019). https://doi.org/ 10.1103/PhysRevD.100.045009. arXiv:1905.05101 [hep-th]

30. N. Bjerrum-Bohr, A. Cristofoli, P.H. Damgaard and H. Gomez, Scalar-graviton amplitudes. JHEP 11, 148 (2019). https://doi.org/ 10.1007/JHEP11(2019)148. arXiv:1908.09755 [hep-th] 